\title{
Structural properties of contractile gels based on light-driven molecular motors: a small-angle neutron and X-ray study
}

\author{
Giacomo Mariani, ${ }^{a}$ Jean-Rémy Colard-Itté, ${ }^{b}$ Emilie Moulin, ${ }^{b}$ Nicolas Giuseppone, ${ }^{\text {b }}$ and Eric Buhler*a
}

The detailed structure of active polymer gels built by integrating light-driven rotary molecular motors as reticulation units in polymer networks is discussed as a function of gel composition. Upon light-irradiation, the collective rotation of molecular motors is translated into the macroscopic contraction of the gels through polymer chains twisting. The major role of the characteristic ratio $c / c^{*}$ ( $c^{*}$ being the overlap concentration of the polymer-motor conjugates before crosslinking) on the contraction efficiency is exploited. Combined small-angle neutron and X-ray scattering experiments reveal the importance of heterogeneities in the macroscopic contraction process: the mesh size of the network increases under irradiation in the whole range of $c / c^{*}$, an increase that is maximal for $c / c^{*}=1$; i.e. at higher contraction efficiency. Furthermore, the mesh size of the network reaches equilibrium within a short period of time, while the heterogeneiteis increase in size untill the end of the contraction process. Finally, the significant motorized twisting of polymer chains within the network allows to foresee the design of new storage energy systems.

\section{Introduction}

The integration of molecular motors in polymer systems is of particular interest to design stimuliresponsive materials capable of generating out-of-equilibrium functions by mechanical transduction. ${ }^{1}$ First inspiration can be found in nature where muscles integrate biomolecular ${ }^{2,3}$ motors (myosin heads) capable of out-of-equilibrium pulling collective motions into a biopolymers network (actin and myosin filaments) when supplied with adenosine triphosphate (ATP) external energy. ${ }^{4}$ This sliding filament mechanism is amplified up to the macroscopic scale resulting in highly efficient muscular contraction and extension.

The access to synthetic mechanically active materials made of artificial molecular machines represents a current challenge at the interface between chemistry, physics, and nanotechnologies. In particular, various molecular switches working at thermodynamic equilibrium have been integrated into polymer systems in order to amplify their collective nanoactuations across increasing length scales. ${ }^{5,6-21}$ However, the integration of more complex molecular motors, ${ }^{1,5,22,23}$ which are able to produce an increasing work on their environment by functioning out-of-equilibrium, are still very scarce. Using Feringa's light-driven rotary motors ${ }^{24-26}$, we have recently developed motor-polymer conjugates capable of unidirectional rotation under irradiation and acting as dynamic reticulation nodes into polyethylene glycol (PEG)-based chemical gels. ${ }^{27,28}$ Such materials are capable of light-induced contraction at macroscopic scale as schematically presented in Figure 1. Here, UV radiation triggers and sustains the continuous unidirectional rotation of the motors, which in turn twist pairs of polymer chains leading to the global contraction of the entire network. This process is not reversible since the rotation of the motor, and therefore the twisting of the polymer chains, is unidirectional. In a more recent study, a reversible process has been demonstrated by making use of a second population of rotary units (i.e. modulators), capable of turning in the opposite direction of the motors and to play the role of elastic releasers after contraction. In this case, the total work output is controlled by the net flux of clockwise and anticlockwise rotations between the motors and modulators. ${ }^{29}$

Our first mechanical studies of the light-induced contraction process have also revealed an optimal concentration for gel preparation in order to obtain maximal contraction efficiency. ${ }^{30}$ This optimal concentration remarkably corresponds to the critical overlap concentration $\left(c^{*}\right)$ of the motor-polymer conjugates used before crosslinking. In addition, by combining rheology and initial SANS experiments, we have determined a number of important guidelines to design and potentially improve the efficiency of this first generation of motorized active gels. We have shown that the macroscopic contraction is accompanied by an increase of the elastic modulus, which directly results from the increase of the polymer strands density under irradiation. Interestingly, the ratios between elastic modulus values before and after irradiation were approximately equal to the inverse ratios of the volumes measured for the gels before and after contraction. Finally, it was suggested that heterogeneities in the gel play an important role in the efficiency of the contraction of the material. However, several open questions of major interest remain unanswered yet. In particular, it is important to understand how the presence of heterogeneities composed by ineffective polymer strands (i.e. chains between two crosslinks) lowers the contraction efficiency, which is defined by the volume change before and after irradiation. The distribution and the structure of such heterogeneities are also unknown, as well as their role on 
the material contraction and their impact on the efficiency of the process. Furthermore, a complete description of their behavior and of the evolution of the network correlation length (i.e., mesh size of the network) with irradiation time is still completely missing.

In an attempt to clarify the above issues, we performed an extended structural investigation at different concentrations of gel preparation (that is chemical cross-linking) ranging from $c<<c^{*}$ to $c>>c^{*}$ and for different light-irradiation times using combined small-angle neutron (SANS) and X-ray scattering (SAXS) experiments. We fully established the major importance of the $c / c^{*}$ parameter for macroscopic contraction efficiency, which can be tuned by changing the polymer chain length and/or the concentration and which was determined through a detailed structural characterization of the polymermotor conjugates before crosslinking. In particular, we identified four contraction regimes for $c / c^{*}$ values spanning both $c / c^{*}<1$ and $c / c^{*}>1$ domains. Additionally, combination of SANS and SAXS, two complementary techniques with different contrast terms and respectively sensitive to the polymer network or to the whole structure, enabled us to track changes with irradiation time in the mesh of the polymeric network and in heterogeneities. Counter-intuitively, the mesh of the network increases under irradiation for the whole range of $c / c^{*}$ values. This increase is maximal for $c / c^{*}=1$; i.e., for the higher contraction efficiency. Furthermore, for $c / c^{*}=1$, the mesh of the network remains constant in size after $3 \mathrm{~h}$ of irradiation, while the size of heterogeneities further increases until the maximum contraction is reached after $15 \mathrm{~h}$ of irradiation. Finally, the motorized twisting and entanglement of efficient polymer strands within the network is found to be particularly significant, which is of fundamental importance for the design of storage energy active materials based on the integration of nano-machines within main polymer networks.
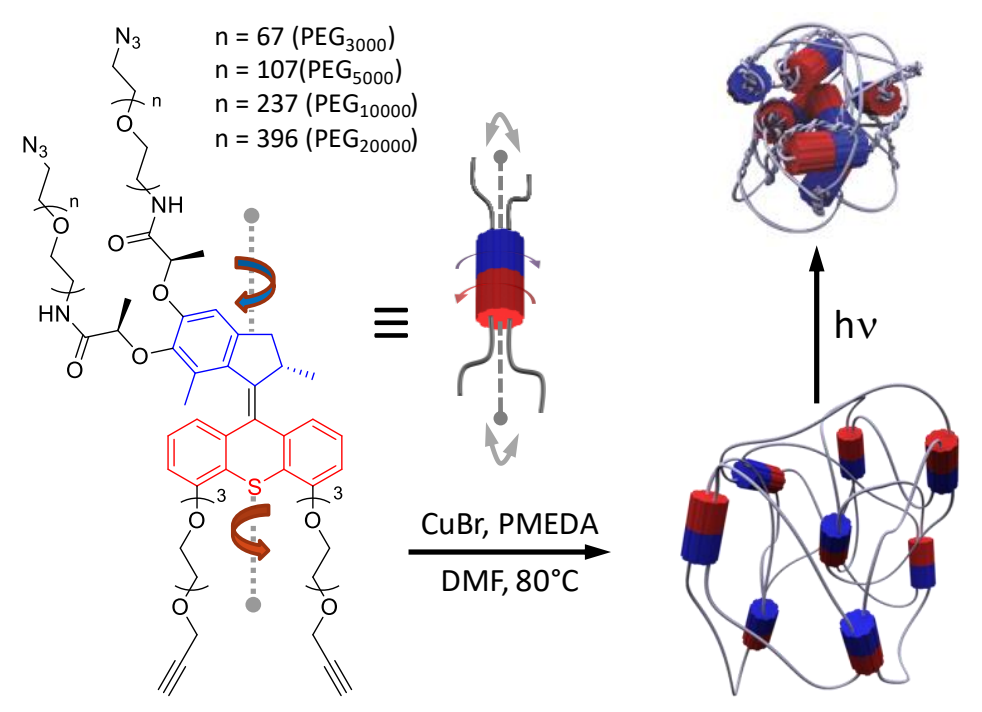

Figure 1. Schematic representation of chemical gels (bottom right) cross-linked by light-driven rotary motors (blue and red cylinders represent the branched motor units with their extended chemical structures shown on the left). By cycling unidirectional rotation of the molecular motors with UV light, the twisting of pairs of neighboring PEG polymer chains leads to a contraction of the entire polymer network up to macroscopic scale (top right).

\section{Materials and Methods}

\section{Materials and crosslinked gel preparation}

All reagents and solvents have been purchased at the highest available commercial quality and used as received (Sigma Aldrich, Acros and TCI). $N_{3}-P E G_{x}-N_{2}$, with various PEG chain length (i.e. molecular weight $x$ ), have been purchased from Iris Biotech. PEG chain molecular weight ranges from 3000 to $20000 \mathrm{~g} \mathrm{~mol}^{-1}$, and precisely: $\mathrm{N}_{3}-\mathrm{PEG}_{3000}-\mathrm{NH}_{2}\left(M_{\mathrm{w}}=3073 \mathrm{Da}\right), \mathrm{N}_{3}-\mathrm{PEG}_{5000}-\mathrm{NH}_{2}\left(M_{\mathrm{w}}=4790\right.$ and $5200 \mathrm{Da}), \mathrm{N}_{3}-\mathrm{PEG}_{10000}-\mathrm{NH}_{2}\left(M_{w}=10526 \mathrm{Da}\right)$ and $\mathrm{N}_{3}-\mathrm{PEG}_{20000}-\mathrm{NH}_{2}\left(M_{w}=17479 \mathrm{Da}\right)$, where $M_{w}$ is the weight-averaged molecular weight. Motors have been synthesized using literature protocols. ${ }^{27,30}$ The synthesis and purification of the motor-PEG conjugates is described in the literature as well. ${ }^{27}$ To prepare the gels, a cross-linking process obtained by the "click" $\mathrm{Cu}(\mathrm{I})$-catalyzed Huisgen 1,3-dipolar cycloaddition, was used. ${ }^{27}$ Gels have been prepared in a homemade metal mold $(2 \mathrm{~cm} \times 2 \mathrm{~cm} \times 458$ 
$\mathrm{m})$. The motor-PEG conjugated has been first dissolved in DMF. In a second phase, 2 eq. of $\mathrm{CuBr}$ and of Pentamethyldiethylenetriamine PMDETA have been added to the motor-PEG conjugate solution and the newly obtained solution moved to the mold. The gel has been then obtained heating the mold up to $80^{\circ} \mathrm{C}$ in a sand path for $30 \mathrm{~min}$ and cooled down to room temperature for another 30 min. The formed gel has been repetitively washed with EDTA, EtOH and water and then brought into toluene by using acetonitrile.

Gel irradiation studies were conducted in dry, degassed toluene. The gel was swollen in a glass vial filled with toluene. All gel manipulations were performed in a room with fluorescent light bulbs emitting over $400 \mathrm{~nm}$ so as not to activate the rotation of the motor. The sample was placed $1 \mathrm{~cm}$ above a $6 \mathrm{~W}$ UV lamp (to avoid any heating) equipped with a tube centered around $365 \mathrm{~nm}$ (maximum of absorption of the motor), with a camera on top of the vial. The contraction process of the gels by UV-light irradiation at $\square 365 \mathrm{~nm}$ has been followed by taking screenshot of the surface at different times. The relative percent volume at elapsed time $t$ has been estimated comparing the initial surface area $S$ and the one at time $t, S_{t}$, and the formula $\%=\left(S_{t} / S_{i}\right)^{n}$ with $n=3 / 2$ as for an isotropic contraction. This isotropic contraction was verified on several samples before assuming it is true for all of them. We measured the change in thickness of the film using a micrometer screw gauge mounted on a piezoelectric rheometer. ${ }^{30}$ The thickness was determined for the minimum distance leading to a measurable rheological effect.

\section{Small Angle Neutron Scattering (SANS)}

SANS experiments ${ }^{31}$ were performed on the D11 beamline at Institut Laue-Langevin at Grenoble (ILL, France) $)^{31-34}$ and on the PACE beamline at the Léon Brillouin Laboratory at Saclay (LLB, France). At ILL, an incident wavelength $\lambda$ of $6 \AA$ was used for 3 sample-to-detector distances $(1.2,8$ and $40 \mathrm{~m})$, allowing to access a total range for the magnitude of the scattering vector $q$ varying between $0.003 \AA^{-}$ 1 and $0.3 \AA^{-1}$. $^{31-34}$ At LLB, two different wavelengths $\lambda$ (13 and $4.6 \AA$ ) and 3 sample-detector distances were used: 1,3 and $4.7 \mathrm{~m}$. A total scattering vector range of $0.004 \leq q\left(\AA^{-1}\right) \leq 0.3$ was thus investigated. The scattering vector is defined by $q=4 \pi / \lambda \sin (\theta / 2)$, where $\theta$ is the scattering angle. Data were corrected for empty cell scattering, electronic background and detector response and then converted to absolute scale $\left(\mathrm{cm}^{-1}\right)$ using normalization by the attenuated direct beam classical method.

For a polymer solution we usually write the scattered intensity $I(q)\left(\mathrm{cm}^{-1}\right)$ as:

$I(q)=\frac{1}{V} \frac{d \sigma}{d \Omega}=k^{2} c N_{a} g(q, c)=k^{2} c N_{a}\left[g_{1}(q)+c g_{2}(q)\right]$

$V\left(\mathrm{~cm}^{-3}\right)$ is the sample volume and, $d \sigma / d \Omega\left(\mathrm{cm}^{2}\right)$, the scattering cross-section. $g_{1}(q)=N P(q)$ is a dimensionless intramolecular term, where $P(q)$ is the chain form factor such as $P(0)=1$ and $N$, its degree of polymerization. $\mathrm{cg}_{2}(q)$ is a dimensionless intermolecular term. $k^{2}\left(\mathrm{~cm}^{2}\right)$ represents the contrast factor; $c$ (in mol. $\mathrm{cm}^{-3}$ ), the monomer concentration; $\mathrm{Na}_{\mathrm{a}}\left(\mathrm{mol}^{-1}\right)$, the Avogadro's number. The scattered intensity could also be written as:

$I(q)=k^{2} c N_{a} g_{1}(q)\left[1+c \frac{g_{2}(q)}{g_{1}(q)}\right]=k^{2} c N_{a} g_{1}(q) S(q)$

allowing to define a dimensionless effective structure factor: $S(q)=1+c g_{2}(q) / g_{1}(q)$.

By introducing, $\Delta \rho^{2}=\left(\rho_{\text {monomer }}-\rho_{\text {solvent }}\right)^{2}\left(\mathrm{~cm}^{-4}\right)$, the difference in scattering length density between polymer and solvent, instead of the contrast factor $k^{2}, V_{P}\left(\mathrm{~cm}^{3}\right)$ the dry volume of the polymer, instead of the degree of polymerization $N$, and the monomer volume fraction $\phi$ instead of the monomer concentration c, we can still write: ${ }^{31}$

$I(q)=\frac{1}{V} \frac{d \sigma}{d \Omega}=\Delta \rho^{2} \phi V_{P} P(q) S(q)$

The scattering length densities of the monomer, $\rho_{\text {monomer }}$, and that of the solvent molecule, $\rho_{\text {solvent }}$, are determined from their known chemical compositions, using the relation:

$\rho=\sum n_{i} b_{i} / N_{a} m v$

where $b_{\mathrm{i}}$ is the scattering length of the $n_{\mathrm{i}}$ atoms of the monomer or the solvent molecule; $m$ and $v$, the molar mass and specific volume of the monomer or the solvent molecule, respectively. The specific volumes of the PEG monomers and rotary motors have been taken to be equal to $1 / 1.126=0.89$ and $1 / 1.10=0.91 \mathrm{~cm}^{3} \mathrm{~g}^{-1}$, respectively. ${ }^{27}$ That of the solvent is $1.06 \mathrm{~cm}^{3} \mathrm{~g}^{-1}$ for deuterated toluene; $1.15 \mathrm{~cm}^{3}$ $\mathrm{g}^{-1}$ for hydrogenated toluene.

\section{Small Angle X-ray Scattering (SAXS)}


The synchrotron radiation X-ray scattering data were collected on the SWING beamline at Soleil Synchrotron Facility in Saint-Aubin, France. The incident energy was $12 \mathrm{keV}$. The sample-detector (Aviex CCD) distance was varied between 1.5 and $3.6 \mathrm{~m}$ to cover a $q$ range from 0.003 to $0.6 \AA^{-1}$. Typically, 10 successive frames of $0.5 \mathrm{~s}$ each were recorded for both solvent and sample. It was verified that $\mathrm{X}$-ray did not cause irradiation damage by comparing the successive spectra. Each frame was first angularly averaged over all frames and the pure solvent spectrum was subtracted. Intensities were scaled using the scattering of water. In the case of SAXS, the scattering length density can be calculated by replacing $b_{i}$ with $Z_{i} r_{e}$ in eq. 4 , where $r_{e}=2.8110^{-13} \mathrm{~cm}$ is the classical radius of the electron and $Z_{i}$ is the atomic number of the $i^{\text {th }}$ atom.

\section{Comparison between SANS and SAXS contrast}

For such gels, it is usual to consider a mixed structure based on a semi-dilute or concentrated polymer solution with some immersed rotary motor junctions, which are cross-links or cross-link aggregates. The contribution to the scattered intensity of each part of the structure (polymer or motor) depends on the radiation through the contrast term $\left(\Delta \rho^{2}\right.$ polymer or $\Delta \rho^{2}$ motor $)$. Hence, the SANS and SAXS experiments can lead to distinct results. The scattering length densities calculated for the solvent, the motorpolymer conjugates as well as for the different chemical parts composing the conjugates; i.e., motors and polymer chains, are given in Table 1 . Inspection of the contrast values, $\Delta \rho^{2}$, clearly indicates that the signal is mostly arising from the PEG chains in SANS experiments. The PEG chains and the central part of the rotary motors have closer contrast terms in the case of SAXS, indicating that this technique is more sensitive to the overall structure than SANS. Therefore, the two techniques give different and complementary structural information and it is crucial to combine them to access the complete structural picture of our chemically crosslinked gels.

\section{Dynamic Light Scattering (DLS)}

The measurements used a 3D DLS spectrometer (LS Instruments, Fribourg, Switzerland) equipped with a $25 \mathrm{~mW} \mathrm{HeNe}$ laser (JDS uniphase) operating at $\lambda=632.8 \mathrm{~nm}$, a two channel multiple tau correlator (1088 channels in

autocorrelation), a variable-angle detection system, and a temperature controlled index matching vat (LS Instruments). The scattering spectrum was measured using two single mode fiber detections and two high sensitivity APD detectors (Perkin Elmer, model SPCM-AQR-13-FC). Fluctuations in the scattered intensity with time $I(q, t)$ (also called count rate), measured at a given scattering angle, $\theta$, or equivalently at a given scattering wave vector, $q=(4 \pi n / \lambda) \sin (\theta / 2)$, are directly reflecting the so-called Brownian motion of the scattering particles (refractive index $n=1.49$ at $20^{\circ} \mathrm{C}$ for toluene).

In dynamic light scattering (DLS), ${ }^{35,36}$ the fluctuation pattern is translated into the normalized time autocorrelation function of the scattered intensity:

$g^{(2)}(q, t)=\frac{\langle I(q, 0) I(q, t)\rangle}{\langle I(q, 0)\rangle^{2}}$

For a diffusive process with a characteristic time inversely proportioned to $q^{2}, g^{(2)}(q, t) \sim \exp \left(-2 D q^{2} t\right)$, with $D$ the mutual diffusion coefficient. The Stokes-Einstein relation permits to determine the hydrodynamic radius, $R_{\mathrm{h}}$, of the scattered objects; $R_{\mathrm{h}}=\mathrm{k} T / 6 \pi \eta D$, if the temperature $T$ and solvent viscosity $\eta$ are known (here $\eta=0.59 \mathrm{cP}$ at $20^{\circ} \mathrm{C}$ for toluene). The size distribution was determined using the classical cumulant method and the CONTIN algorithm based on the inverse Laplace transform of the correlation function.

Table 1. Densities, SANS and SAXS scattering length densities and contrasts per unit volume of the total motor-polymer conjugate, the $\mathrm{PEG}$ chain, the central unit of the rotary motor, and solvent. The total chemical composition is given for a $\mathrm{M}_{\mathrm{W}, \mathrm{PEG}=5000 \mathrm{~g} \mathrm{~mol}}{ }^{-1} \mathrm{sample}$.

\begin{tabular}{|c|c|c|c|c|c|c|}
\hline & $\begin{array}{c}\text { Density } \\
g \mathrm{~cm}^{-3}\end{array}$ & $\begin{array}{c}\text { SLD } \\
10^{-6} \AA^{-2}\end{array}$ & $\begin{array}{c}\Delta \rho_{\text {SANS }}^{2} \\
10^{-11} \AA^{-4}\end{array}$ & $\begin{array}{c}\text { SLD } \\
10^{-6} \AA^{-2}\end{array}$ & $\begin{array}{c}\Delta \rho_{S A X S}^{2} \\
10^{-11} \AA^{-4}\end{array}$ & Chemical Composition \\
\hline Total & 1.1 & 0.711 & 2.45 & 10.185 & 0.474 & $\mathrm{C}_{540} \mathrm{H}_{1040} \mathrm{O}_{256} \mathrm{~N}_{8} \mathrm{~S}$ \\
\hline $\begin{array}{c}\text { PEG chain } \\
\begin{array}{c}\text { Central part of } \\
\text { rotary motor }\end{array}\end{array}$ & 1.126 & -0.492 & 3.79 & 10.822 & 0.792 & $\mathrm{C}_{2} \mathrm{H}_{6} \mathrm{O}$ \\
\hline d-Toluene & 0.943 & 1.907 & 1.41 & 9.841 & 0.34 & $\mathrm{C}_{30} \mathrm{H}_{26} \mathrm{O}_{4} \mathrm{~N}_{2} \mathrm{~S}$ \\
\hline
\end{tabular}




\section{Results and Discussion}

To understand the major role played by heterogeneities and chain conformations on the efficiency of the contraction process, and therefore to gain insights on the mechanisms regulating the contraction, several light-driven molecular motors-based gels have been prepared by independently varying the number or mass concentration of motor-polymer conjugates; i.e., number or mass concentration of chemical crosslinks, and the polymer chain length (see details in Table 2). The central rotary part, which is the network reticulation unit, is identical for all conjugates. This results in a change of the concentration across the overlap concentration $c^{\star}$ that has been identified as the crucial parameter regulating the contraction efficiency. ${ }^{30}$ Therefore as first step, the $c^{*}$ concentration needs to be evaluated for each motor-polymer conjugate systems. This was achieved by determining the structural parameters of such conjugates in the dilute regime before the chemical crosslinking process.

\section{Motor-polymer conjugates characterization in dilute regime before chemical crosslinking}

The structural properties of the motor-polymer conjugate solution have been studied, before polymer crosslinking by click chemistry, using combined light and small angle neutron scattering in the dilute regime, i.e. at a concentration equal to $c=2 \mathrm{mM}$ for which conjugates are distant and do not overlap. The SANS spectra obtained for two motor-polymer conjugates formed from motors functionalized by two PEG chains with individual molecular weights equal to 4790 and $5200 \mathrm{~g} \mathrm{~mol}^{-1}$, namely $\mathrm{PEG}_{4790}$ and $P E G_{5200}$, are reported in Figure 2. Despite some differences, both curves present a similar overall behavior that can be described by the following sequence: a low-q upturn in which the signal increases as $q^{3}$ and indicates that conjugates form aggregates larger than the available $q$ window (i.e. size larger than $40 \mathrm{~nm}$ ), mostly due to a lower solubility of the motors in toluene. Interestingly, this motor aggregation may be the cause of heterogeneities formation during the gel crosslinking process (as discussed in the next parts). A variation similar to a Guinier law associated with the finite mass and size of the motor-polymer conjugates is visible in the intermediate range of $q$ values. Finally, in the high $q$ domain, the two consecutive $q^{5 / 3}$ and $q^{1}$ power laws arise from the signal of the polymer PEG chains expanded in good solvent. The $q^{*}$ value at which the $q^{5 / 3}-q^{1}$ transition takes place gives an estimate for the PEG chain persistence length using $l_{p} \sim 1.91 / q^{*} \sim 1.1 \mathrm{~nm}{ }^{37,38}$ and characterizes the crossover between the coil-like and rod-like behavior of the polymers. It is important to note that the SANS signal at high $q$ mostly arises from the PEG chain according to the contrast values given in Table 1.

The data in the intermediate $q$ regime have been fitted using a classical Guinier approximation:

$I(q)=I_{0} e^{-\frac{q^{2} R_{g}^{2}}{3}}$

Giving the radius of gyration, $R_{\mathrm{g}}$, of the motor-polymer conjugates. The best fit of the data using equation 6 gives (continuous lines in Figure 2) $R_{g}=3.4 \pm 0.1$ and $4.3 \pm 0.2 \mathrm{~nm}$ for PEG arm molecular weights equal to 4790 and $5200 \mathrm{~g} \mathrm{~mol}^{-1}$, respectively. Radius of gyration values are in line with dynamic light scattering measurements displaying a main cooperative diffusion relaxation giving a hydrodynamic radius $R_{\mathrm{h}}$ equal to $3.35 \pm 0.07 \mathrm{~nm}$ for the $\mathrm{PEG} \mathrm{G}_{5200}$ conjugates and a $\rho=R_{\mathrm{g}} / R_{\mathrm{h}}$ ratio of $\sim 4.3$ / $3.35 \sim 1.3$; i.e., a value in agreement with that derived for wormlike chains and equal to 1.5. A slow mode of small amplitude corresponding to a minority population of larger aggregates is also visible in the long-time range of the correlation function in accordance with the $q^{3}$ low- $q$ upturn observed in the SANS profiles. 


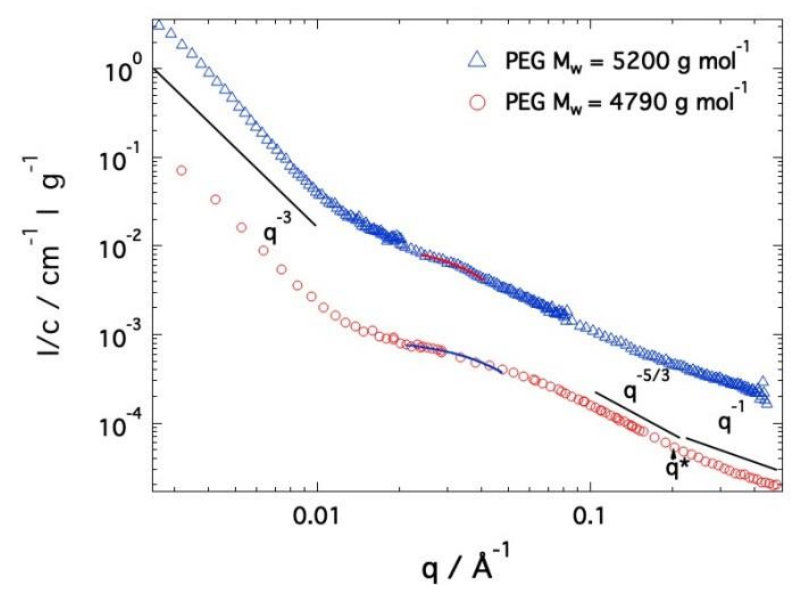

Figure 2. Scattering profiles of dilute solutions of motor-polymer conjugates before the click reaction. $P E G_{4790}$ curve is vertically shifted downwards for clarity. The continuous lines represent the best fits of the data using a Guinier model. Concentrations are 43.6 and $58 \mathrm{~g} / \mathrm{l}$ for PEG arms molar mass equal to 4790 and $5200 \mathrm{~g} \mathrm{~mol}^{-1}$, respectively. The molar concentration is equal to 5 $\mathrm{mM}$.

Motor-polymer conjugates begin to overlap when their concentration exceeds the overlap concentration, $c^{\star}$. This crossover concentration separating dilute and semi-dilute regimes, where chains interpenetrate and form a physical network, decreases with the chain length (or equivalently the degree of polymerization $N$ ) with $c^{\star} \sim N^{4 / 5}$ in good solvent and can be estimated by using the radius of gyration $R_{\mathrm{g}}$ of the motor-conjugates in dilute regime: ${ }^{39}$

$c^{*}=\frac{M}{\frac{4}{3} \pi R_{g}^{3} N_{a}}$

Where $N_{a}$ is the Avogadro's number. In eq. 7, $M$ is the total mass of the motor-polymer conjugate. With $R_{\mathrm{g}}=4.3 \mathrm{~nm}$ and $M$ the total molecular weight, one finds a critical overlap concentration $c^{*}=55 \mathrm{~g} / \mathrm{L}$ for $\mathrm{PEG}_{5200}$, which corresponds to a molar concentration of conjugates equal to $5.3 \mathrm{mM}$. For conjugate PEG 4790 with $R_{\mathrm{g}}=3.4 \mathrm{~nm}$, one obtains a value for $c^{\star}$ equal to $57 \mathrm{~g} / \mathrm{l}$, which is a very similar value.

These values can be scaled to other motor-polymer conjugate molecular weights using the dependence of $c^{*}$ with $N$ calculated for linear polymers in good solvent, ${ }^{39}$ which are characterized by a $\rho$-ratio of the same order of that determined above:

$c_{2}^{*} \sim N^{-4 / 5}=c_{1}^{*}\left(\frac{M_{2}}{M_{1}}\right)^{-4 / 5}$

This allows an estimate of the overlap concentration for each studied motor-polymer conjugate. All values are collected in Table 2.

\section{Effect of irradiation on the swelling and de-swelling properties of motor-polymer conjugate crosslinked-} gels

All experiments were performed with fully swollen gels at room temperature in toluene. Three different series of samples have been analyzed. We first explored the effect of the polymer length on the swelling properties of the materials.

Different gels were formed from motors functionalized by PEG chains with molecular weights varying between 3000 and $20000 \mathrm{~g} / \mathrm{mol}$, namely $P E G_{3000}, P E G_{5000}, P E G_{10000}$ and $P E G_{20000}$. To prepare these gels, the initial concentration of polymer-motor conjugates, which is the concentration used before the cross-linking process obtained by the "click" $\mathrm{Cu}(\mathrm{I})$ - catalyzed Huisgen 1,3-dipolar cycloaddition, was fixed at $c=5 \mathrm{mM}$. This implies that all chemically crosslinked gels are composed by the same number of motors and polymer strands, making the polymer contour length between motor-like crosslinks the only variable parameter (i.e. length at maximum possible extension). A polymer strand is a chain or a chain part between two crosslinks.

In a second set of preparations, different gels were prepared by varying the motor initial concentration between 4 and $10 \mathrm{mM}$ and by keeping the polymer mass constant to $M_{\mathrm{w}}=5000 \mathrm{~g} / \mathrm{mol}$ (i.e. the PEG length between crosslinks). 
In the last set of experiments, the PEG molecular weight has been varied keeping constant the motor mass concentration in $\mathrm{g} / \mathrm{l}$. This allows a direct observation of the effect of the polymer strand volume fraction.

It is important to distinguish the polymer volume fraction in the state of gel network preparation and the one in the gel state of interest. Here, all the studied gels are at equilibrium and are thus fully swollen with solvent. The de-swelling behaviour is obtained by light irradiation and by the motor rotation that enforces entanglements and induces the contraction of the material. The details of the sample composition as well as their volume changes obtained under irradiation are reported in Table 2. Its inspection clearly shows that volume changes and, therefore, contraction efficiencies (i.e. maximum variations of gel volume before and after irradiation) are dependent on the gel composition. An example is given in Figure 3 showing photographs taken during the contraction process for a $5 \mathrm{mM}$ PEG5000 gel over two hours of UV irradiation.

To allow a better comparison of the data of the different series of gel preparation, the universal parameter $c / c^{*}$, representing the ratio between the polymer-motor conjugate concentration and the overlap concentration, was introduced. The latter has been determined in the previous part in the dilute regime for each sample using equations 7 and 8.
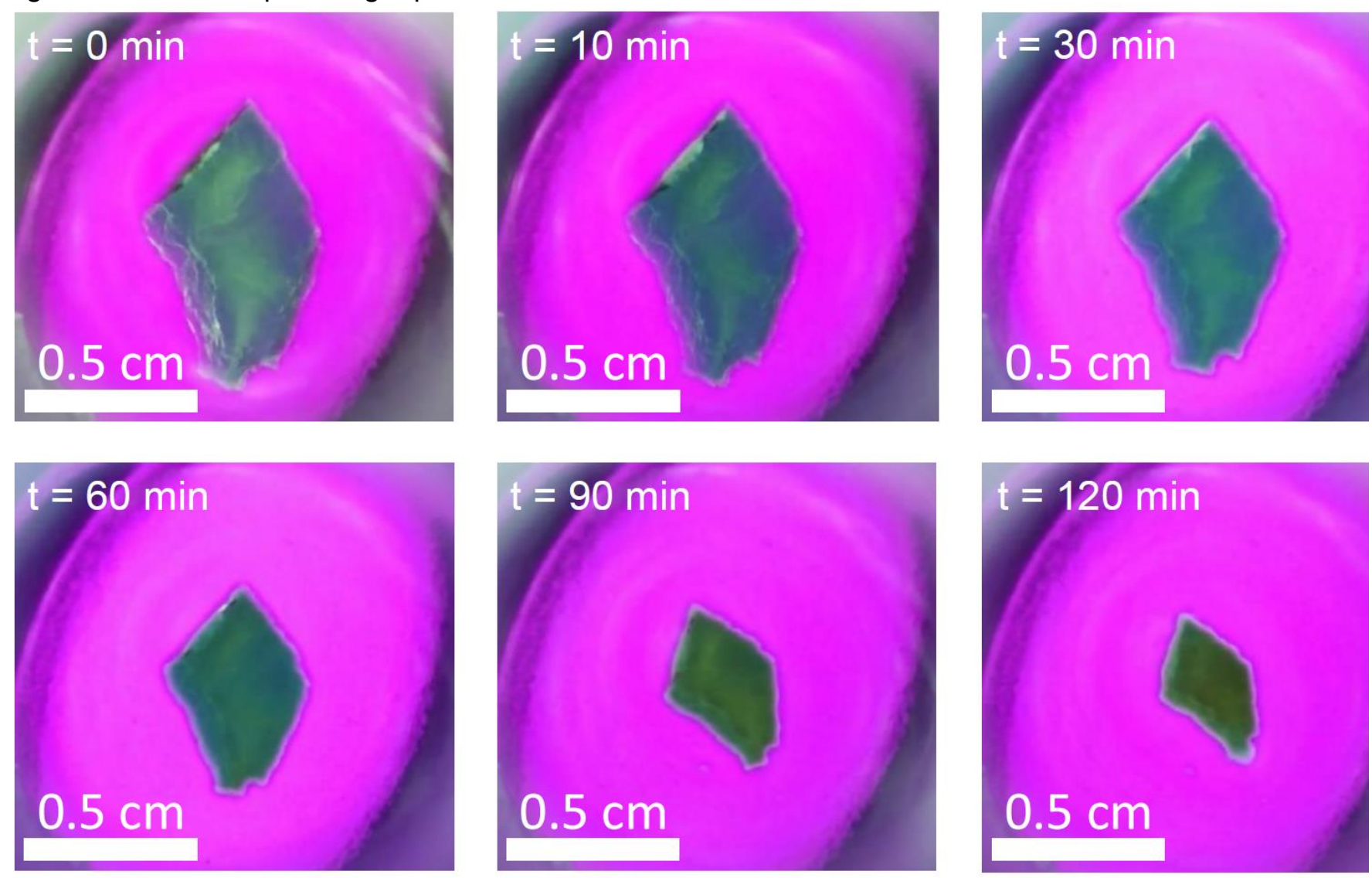

Figure 3. Typical contraction of a $1 \mathrm{~cm}^{2}$ piece of gel observed over a 2 hours UV irradiation. The gel contraction process was followed by video and stopped after 2 hours. The motor-polymer conjugate concentration was $5 \mathrm{mM}$ and the PEG mass 5000 $\mathrm{g} / \mathrm{mol}$.

This parameter permits comparison of the results according to the degree of interpenetration of each system. All sets of experiment can thus be combined in a master curve by plotting the contraction rate $V_{i} / V_{f}$ as a function of $c / c^{*}$, where $V_{i}$ and $V_{f}$ are the volumes before and after irradiation, respectively (see Figure 4). The most efficient contraction $\left(V_{i} / V_{f}^{\max }=3\right.$ ) takes place when $c / c^{*}=1$ (obtained for 5 $\mathrm{mM}$ motor concentration and for $\mathrm{PEG}_{5000}$ ) and reveals the major role played by the overlap concentration $c^{*}$. When $c / c^{*}<1$, the contraction efficiency is well reduced until the concentration is too low to form a gel system (i.e. motor-polymer conjugates being too distant). Below $c^{*}$ the efficiency of the click reaction is reduced and the gel is not fully cross-linked resulting in a decrease of polymer chains winding and thus of its contraction efficiency. When $c / c^{*}>1$, the contraction efficiency decreases until the system cannot contract anymore. This is observed for $c / c^{*}>2$, where only a volume variation of $1 \%$ is reported, which is within the error bar. Above $c^{\star}$, the system is denser as it includes 
additional entanglements. This becomes detrimental to the winding of the chains under motor rotation and therefore, the efficiency of the contraction is strongly reduced. At the same time, it has been shown that these additional physical cross-links or entanglements participate in increasing the elastic modulus of the material $G$ ' before contraction. ${ }^{30}$

Table 2. Characteristics of the crosslinked polymer-motor conjugate gels swollen in toluene. $c$ is the polymer-motor conjugate concentration in $\mathrm{g} / \mathrm{l} ; c^{*}$ is the overlap concentration, and $V_{\mathrm{i}} / V_{\mathrm{f}}$ the variation of the volume of the gel ( $V_{\mathrm{i}}$ is the volume before irradiation and $V_{\mathrm{f}}$ the volume after irradiation). Values are obtained after an irradiation time of $180 \mathrm{~min}$. Samples denoted by an

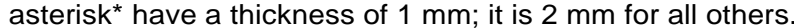

\begin{tabular}{|c|c|c|c|c|c|c|c|}
\hline$M_{w}(\mathrm{PEG})$ & \multicolumn{2}{|c|}{$\begin{array}{c}\text { Polymer-motor conjugate } \\
\text { concentration } c\end{array}$} & $C^{*}$ & $c / c^{*}$ & $\begin{array}{c}\text { Contraction } \\
\text { efficiency }\end{array}$ & $\begin{array}{l}\text { Volume } \\
\text { ratio } V_{\mathrm{i}} / V_{\mathrm{f}}\end{array}$ & $\begin{array}{c}\text { Macroscopic } \\
\text { behavior }\end{array}$ \\
\hline $\mathrm{g} / \mathrm{mol}$ & $g / l$ & $\mathrm{mM}$ & $g / l$ & & $\%$ & & \\
\hline 5000 & 22 & 2 & 55 & 0.40 & - & - & Solution \\
\hline 3000 & 34 & 5 & 79 & 0.43 & 25 & 1.3 & Gel \\
\hline 5000 & 43 & 4 & 55 & 0.78 & 28 & 1.4 & Gel \\
\hline $5000^{*}$ & 43 & 4 & 55 & 0.78 & 50 & 2 & Gel \\
\hline 5000 & 55 & 5 & 55 & 1 & 52 & 2.1 & Gel \\
\hline $5000^{*}$ & 55 & 5 & 55 & 1 & 72 & 3 & Gel \\
\hline 4790 & 73 & 5 & 57 & 1.28 & 36 & 1.6 & Gel \\
\hline 10000 & 55 & 2.75 & 32 & 1.71 & 33 & 1.5 & Gel \\
\hline 5000 & 100 & 9.2 & 55 & 1.8 & 33 & 1.5 & Gel \\
\hline 5000 & 108 & 10 & 55 & 1.97 & 23 & 1.3 & Gel \\
\hline 5000 & 130 & 12 & 55 & 2.36 & 1.1 & 1.01 & Gel \\
\hline 20000 & 55 & 1.5 & 19 & 2.9 & 0 & 1 & Gel \\
\hline 10000 & 104 & 5 & 32 & 3.25 & 1.1 & 1.02 & Gel \\
\hline
\end{tabular}

Results presented in Figure 4 are of major importance since these unambiguously confirm that $c / c^{*}$ is the crucial parameter regulating the contraction, which can be tuned by modifying important polymer parameters such as length, flexibility or concentration. Moreover, the efficiency of this novel class of materials is directly dependent on the sample density: too dense gels cannot contract efficiently or cannot contract at all, while very low density gels are able to contract but with a lower efficiency.

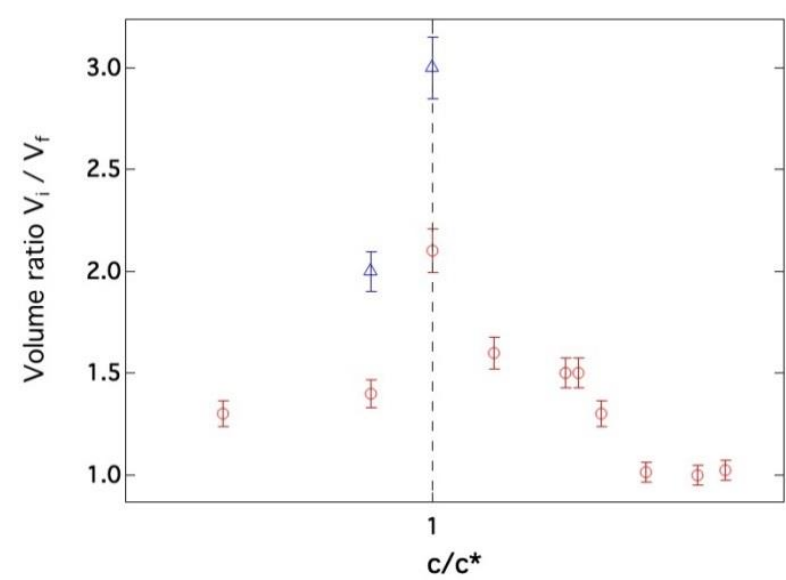

Figure 4. Contraction rate $V_{i} / V_{f}$ as a function of $c / c^{*}$ measured after 180 minutes of UV irradiation. Volumes correspond to the maximum swelling in toluene, $V_{\text {eq. }}$. Sample thickness is $1 \mathrm{~mm}$ for the blue triangle points and $2 \mathrm{~mm}$ for the red circles.

As classical gels, our motor-polymer conjugate crosslinked gels swell in toluene until $V_{\text {eq }}$ for which the osmotic and elastic contributions to the free energy are balanced. ${ }^{40}$ For $c / c^{*}=1$, a swelling ratio equal to $Q=V_{\text {eq }} / V_{\text {dry }}=5.4810^{-2} \mathrm{~cm}^{3} / 0.264610^{-2} \mathrm{~cm}^{3}=20.7$ was found for the non-irradiated gel. This is in very good agreement with the theoretical prediction in good solvent for an excluded volume parameter $v \sim 0.3 b^{3}$, where $b$ is the Kuhn length of the polymer chain.

$Q=\frac{V_{e q}}{V_{d r y}}=\frac{1}{\phi_{s}} \approx\left(\frac{v}{b^{3}}\right)^{0.40} N^{0.57} \phi_{0}^{-1 / 4}, 1 / \mathrm{Q}<\phi_{0}<\phi^{\star *}$

In equation $9, V_{\text {dry }}$ is the volume of the material in the dry state (for which $\phi=1$ ), $\phi_{\mathrm{s}}$ the volume fraction in the fully swollen state, $N$ the number of monomers in polymer strands; i.e., the polymerization degree between chemical crosslinks, which is 110 for $\mathrm{PEG}_{5000}, \phi_{0}$ the polymer volume fraction in the 
preparation state after cross-linking and finally $\phi^{* *} \approx v / b^{3}$ denotes the crossover concentration between semidilute and concentrated solutions. Obukhov et al. predicted a scaling relationship between the elastic modulus and $\phi_{\mathrm{s}}$

$G \sim \phi_{S}^{3 v / 3 v-1 \sim \phi_{S}^{2.3}}$

Where $v=0.588$ in good solvent as confirmed by SANS (see Figure 2). ${ }^{39-40}$ Note that this model and the $c^{*}$ theorem predict the identical dependence of $G$ on $\phi_{s .}{ }^{39,40}$ Data reported in ref 34 show this dependency for both irradiated and non-irradiated gels, indicating that they are both in their own equilibrium state (maximum swelling).

\section{Structure of the cross-linked swollen polymer networks as probed by SANS}

Subsequently, after having characterized the efficiency of the de-swelling of the gels, it was essential to establish the correlations between the macroscopic properties and the molecular structural changes associated with such light-driven material contraction. Combined SANS and SAXS experiments are the most suitable techniques to perform such structural characterization since they permit to investigate at the same time the local conformation and the degree of motors aggregation in the molecular and supramolecular scales $(1-40 \mathrm{~nm})$ directly in solution and gel state without any sample modification. To better relate the structural characterization to the contraction efficiency, the results are discussed as a function of the characteristic ratio $c / c^{*}$. SANS results obtained before and after 15 $\mathrm{h}$ irradiation for several characteristic ratios spanning both $c / c^{*}<1$ and $c / c^{*}>1$ regimes are collected in Figure 5. As stated earlier, the contribution to the scattered intensity of each part of the structure (polymer or motor) depends on the radiation, through the contrast term (Table 1). For SANS, the signal mostly arises from the PEG chains and, hence, neutron scattering patterns permit to follow changes in the polymer network matrix induced by rotation and contraction. This is clearly observed in the intermediate and high $q$ regimes that display the characteristic behavior of the excluded volume PEG chains with a characteristic $q^{5 / 3}-q^{-1}$ transition, and a Lorentzian variation associated with the correlation length that is the mesh size of the entangled network. This applies for all $c / c^{*}$ values, before and after irradiation. The $q^{1}$ dependence is characteristic of the rod-like behavior of the chains for distances smaller than the persistence length, $l_{0}$, while the $q^{5 / 3}$ one is typical for a polymer chain expanded in good solvent. The crossover value $q^{*}$ between these dependencies identifies the persistence length of the PEG chains that remains unchanged under irradiation and equal to $I_{p}=1.91 / q^{*}=1.1 \mathrm{~nm} .{ }^{42,43}$ More importantly, extended and contracted curves are superimposed at high $q$ for all $c / c^{*}$ ratios indicating that the local chain conformation remains unchanged under irradiation. On the contrary, the scattered intensity upturn observed in the low- $q$ regime and associated with the presence of large heterogeneities depends on the irradiation for some $c / c^{*}$ ratios. This is evident for $c / c^{*}=1$, where the maximum contraction efficiency is reported (Figure 5d). Moreover, the Lorentzian variation depends on the $c / c^{*}$ ratio and on the irradiation state as well. These dependencies are discussed below as a function of the rate of entanglements through the characteristic $c / c^{*}$ ratio and of the irradiation:

i) $c / c^{\star}<0.4$. Below $c^{*}$ the efficiency of the click reaction is reduced and the gel is not fully crosslinked or even not formed. Results obtained for $\mathrm{PEG}_{5000}$ at $c / c^{*}=0.4$, i.e. at a concentration well below the overlap concentration and for which the gel cannot form, are shown in Figure 5 a. Obviously, no contraction is observed. The curves before and after irradiation are markedly similar at high $q$ with a signal corresponding to polymer chains expanded in good solvent and comparable to that of individual dilute conjugates. Instead, the curves differ at low $q$ : before irradiation the curve bends over with a very small increase of the signal, while after irradiation the curve strongly increases. The bending over visible before irradiation can be fitted using a Guinier model giving a radius of gyration equal to $R_{g}=9.6 \pm 0.1 \mathrm{~nm}$, a value larger than that of a single polymer-motor conjugate $\left(R_{\mathrm{g}}=4.3 \mathrm{~nm}\right)$ and indicating the formation of aggregates made of about 11 units when the click reaction takes place. After irradiation, two contributions could be identified: one with $R_{g}=9.4 \pm 0.3 \mathrm{~nm}$ as before and another one with $R_{g}=30 \pm 5 \mathrm{~nm}$. Hence, clusters form upon irradiation from association of small $\sim 9 \mathrm{~nm}$ aggregates or less likely by dissociation of clusters already present in solution before irradiation and larger than the maximum distance available by SANS $(40 \mathrm{~nm})$.

ii) $0.4<c / c^{*}<1$ : As the concentration further increases, the motors are interconnected and form a gel (Figure 5b and 5c). However, in this regime, the partial crosslinking results in a decrease of the rate of entanglements. The gel density is low and therefore the systems contract with lower efficiency, which is limited by the spatial distribution of the motors since the crosslinking 
is lessened. Importantly, the low- $q$ signal associated with the presence of large heterogeneities is similar before and after irradiation.

iii) $c / c^{*}=1$. On the contrary, clear and important changes are observed in the low- $q$ domain under contraction when $c \sim c^{*}$ (see Figures $5 d$ and $5 e$ ). Interestingly, $c^{*}$ is the concentration at which the most efficient contraction is observed. At high $q$, the scattering patterns exhibit the characteristic power laws associated with the local conformation of the expanded PEG chains, while the bending over observed in the mid $q$ range can be described by a Lorentzian law allowing the determination of a finite size for the network mesh. After irradiation, the signal at high $q$ remains identical indicating that the PEG conformation does not change under contraction. For the contracted system, the excluded volume regime $q^{5 / 3}$ is however extended to much lower $q$ values, indicating that the mesh of the network is much larger. At the same time, the mid- $q$ Lorentzian variation associated with the mesh size of the network extends thus to much lower $q$ values as well. All the changes take place in the low and mid $q$ regions. At $c$ $=c^{*}$ the system's composition is optimal and the balance between density and sample composition (i.e. motor distribution) leads to the most efficient contraction process. Therefore, the mesh size of the network changes upon contraction as well as its homogeneity.

iv) Regime $c / c^{*}>1$. The SANS curves for 3 samples at different $c / c^{*}$ ratios are given in Figures $5 \mathrm{f}, 5 \mathrm{~g}$, and $5 \mathrm{~h}$. In the $c / c^{*}>1$ regime, the system is denser and includes additional entanglements that are detrimental for the winding of the chains under irradiation and no significant contraction takes place for samples with $c / c^{*}>2$ (Figures $5 \mathrm{~g}$ and $5 \mathrm{~h}$ ). Concomitantly, their corresponding spectra before and after irradiation are obviously identical and show the typical scattering pattern of gels described above. At low $q$, the signal increases as $q^{3}$ for both curves and coincides with the non-ergodic nature of gels (non-homogeneous materials) due to network regions with higher concentration of motors and PEG chains. The presence of such heterogeneities is clearly not affected by the light-induced motor rotation in this rate of entanglements regime. On the other hand, the Lorentzian variation displays some changes (Figures $5 f$ and $5 \mathrm{~g}$ ).

To understand quantitatively the changes observed for all concentration regimes, the curves have been fitted using a structural model specific for gels. In such model, the total scattering intensity is described by a combination of Gauss and Lorentz-type functions: ${ }^{41,42}$

$I(q)=I_{L}(0) \frac{1}{\left(1+q^{2} \xi^{2}\right)}+I_{G}(0) e^{-q^{2} a_{2}^{2}}+B$

In which $I_{L}(0)$ (Lorentzian) and $I_{G}(0)$ (Gauss) are linear coefficients. $\xi$ is the correlation length in the solution-like part of the gel representing the mesh size of the network (Ornstein-Zernicke variation at mid q). $a_{2}$ is generally associated to the size of regions (Gaussian spatial distribution) whose movement is restricted by the crosslinks, which generate elastic constraints and non-uniformities in the polymer concentration when the gel is swollen. We, here, consider that $a_{2}^{2}=R_{g}^{2} / 3$, allowing determination of the radius of gyration, $R_{\mathrm{g}}$, of the large "static-like" heterogeneities in the low $q$ domain. $B$ is the background.

The most significant parameter describing the contraction process is the polymer network mesh size variation, $\Delta \xi$ : after contraction the mesh size $\xi$ changes in the whole concentration range and the largest increase takes place for $c / c^{*}=1$; i.e., at highest contraction efficiency (see Table 3 and Figures $6 \mathrm{a}$ and $6 \mathrm{~b}$ displaying respectively the mesh size and its variation as a function of $\left.c / c^{*}\right)$. For this particular rate of entanglements, it grows from $5.2 \pm 0.2$ to $9.7 \pm 0.2 \mathrm{~nm}$ under contraction. The increase of the number of entanglements due to polymer winding under motors rotation is thus accompanied by a significant variation of the mesh size of the polymer matrix.

In addition, the change in mesh size when $c \leq c^{*}$ is larger as compared to that observed for $c>c^{*}$, mostly due to the higher density of the gel. 

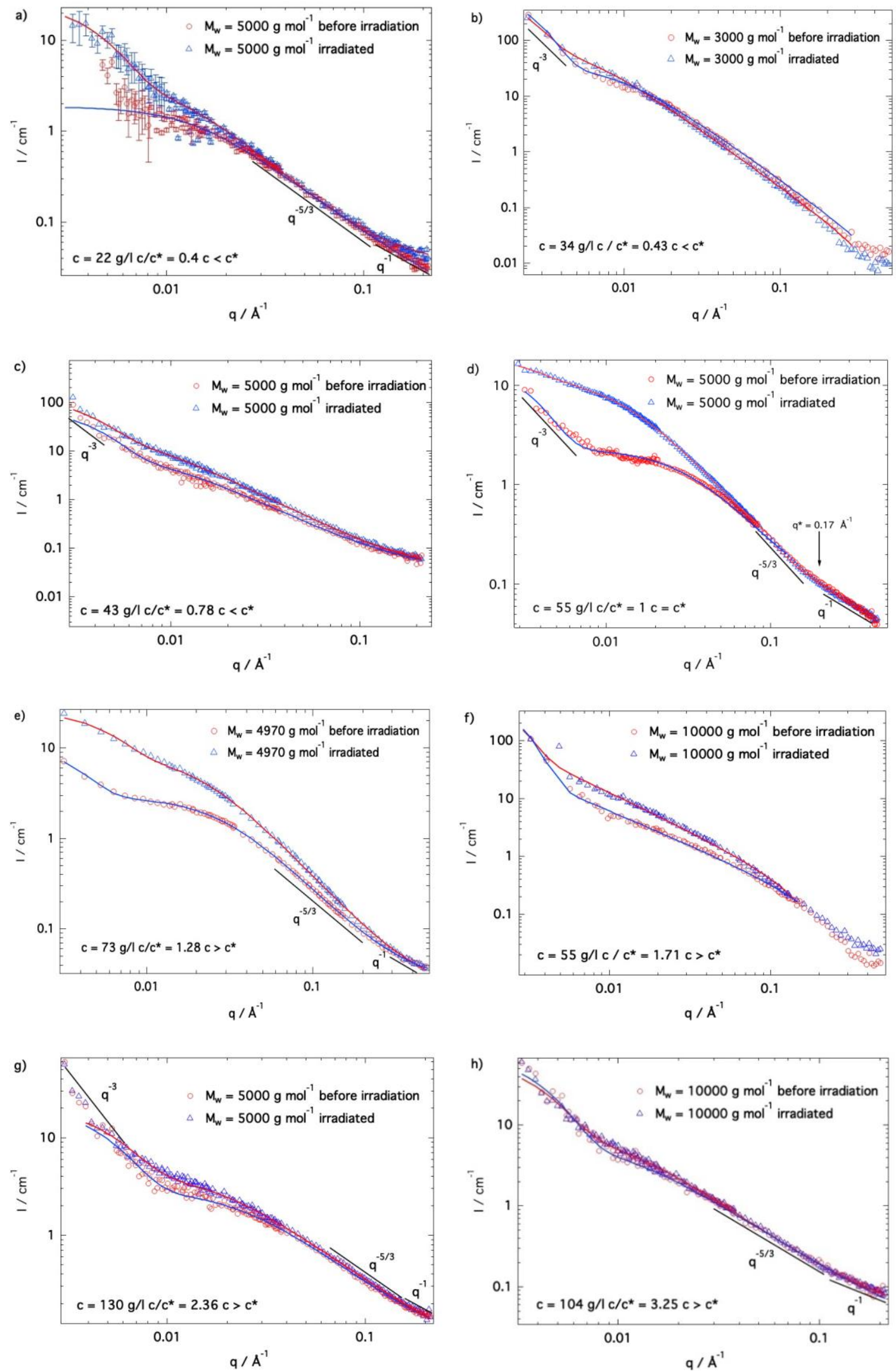

Figure 5. SANS scattering profiles obtained before and after irradiation for $c / c^{*}=0.4$ (a); 0.43 (b); 0.78 (c); 1 (d); 1.28 (e); 17.71 (f); 2.36 (g); and 3.25 (h). The continuous lines represent the fit of the data using eq 11.

When the gel is too dense, the contraction is less effective and so is the increase in mesh size. Another interesting parameter is the Guinier radius of the heterogeneities and its change with contraction (see Table 3). Unfortunately, the size of such heterogeneities is in most cases higher than the available $q$ - 
range; around $60 \pm 15 \mathrm{~nm}$ for instance for $c / c^{*}=1$ before irradiation. The model used to fit the experimental data however gives a size value that can be considered as a lower bound.
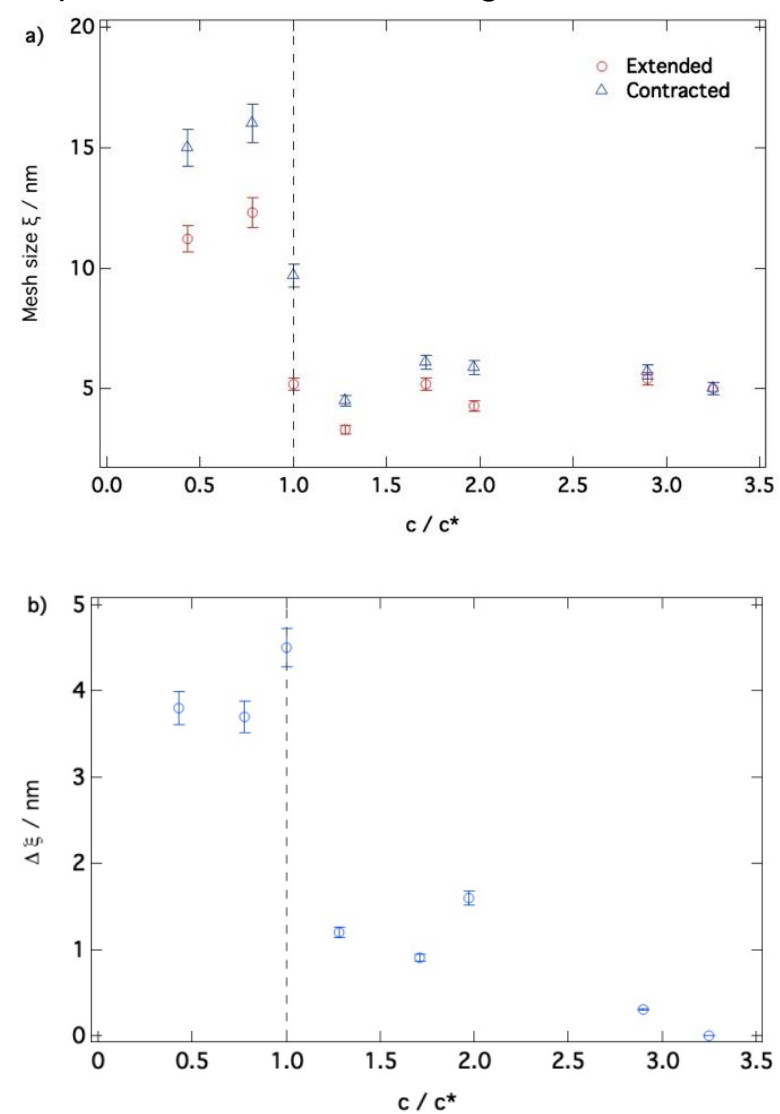

Figure 6. a) Variation of the gel mesh size before and after irradiation with the characteristic $c / c^{*}$ ratio. The change in mesh size is shown in Figure $6 \mathrm{~b}$.

Surprisingly, the signal associated with heterogeneities decreases under motor rotation for concentrations close to the overlap concentration, $c^{*}$, or even disappears at $c=c^{*}$, where maximum efficiency is reached. Indeed, instead displaying an upturn, the irradiated low- $q$ signal exhibits the onset of a plateau and a finite value for the zero- $q$ intensity, which is associated to the finite mass of the correlation length. This may suggest that the PEG chains initially aggregated in heterogeneities are progressively unwound under contraction allowing a reorganization of the polymer network and the formation of a homogeneous gel. Another, most convincing, interpretation is that heterogeneities become much larger under contraction and that the upturn shifts well outside the neutron scattering length scale. To determine which explanation is the most likely, we followed the evolution of the heterogeneities signal and of the network mesh size with intermediate irradiation times at $c / c^{*}=1$ where a significant reorganization of the polymer network is observed.

\section{Effect of irradiation time on the gel structure for $c / c^{*}=1$}

SANS results have revealed that heterogeneities play an essential role in the contraction efficiency of the gel. Formation of heterogeneities may originate from residual motors segregation before crosslinking as shown in Figure 2. This leads to the formation of denser areas, which are initially composed by both PEG and motor aggregates. At $c=c^{\star}$, SANS could either indicate that the polymer network matrix becomes more homogeneous due to the progressive uncoiling of the fraction of PEG chains present in such heterogeneities or, most likely, that heterogeneities are pulled together and thus grow in size. Despite the two different scenarios, SANS shows unequivocally significant network structural changes in the intermediate $q$-range and thus, appears to be the most appropriate technique to follow the correlation length changes with intermediate irradiation times and to find correlations with interesting parameters such as the heterogeneities behavior, the rate of entanglements, the mechanical properties and the contraction efficiency. SANS experiments have been conducted on the powerful D11 beamline at ILL under a fully swollen gel made of PEG ${ }_{5000}$ at $c=c^{*}=55 \mathrm{~g} / \mathrm{l}$ irradiated at various time intervals. Results at different exposure times are shown in Figure 7 . The contraction 
process is investigated step-by-step understanding all the time-dependent changes on the gel structure. After the maximal contraction reached for an irradiation time of $15 \mathrm{~h}$, the gel starts to broke up, presenting macroscopic defects.

We performed two sets of experiments showing the reproducibility of the results (Figures $7 \mathrm{a}$ and $7 \mathrm{~b}$ ). At high $q$, all patterns superimpose perfectly on each other and coincide with the signal of the PEG chains expanded in good solvent with the characteristic $q^{1}-q^{5 / 3}$ transition. Due to the SANS contrast, the signal of the motor units is not visible in the high- $q$ domain. Interestingly, toluene remains a good solvent for all light exposure times and all intermediate contraction states. As the irradiation time increases and the contraction progresses, the Lorentzian and the $q^{5 / 3}$ power laws both extend to much lower $q$ values. This unambiguously indicates that the correlation length associated to the mesh size of the polymer network becomes larger. The shift of the Lorentzian law towards higher scattered intensities is a natural consequence of the extension of the PEG chains signal and thus of the network mesh mass increase. The $q$-value at which the curves bend over and reach the Lorentzian regime changes dramatically with irradiation: from $q=0.035 \AA^{-1}$ before irradiation to $0.015 \AA^{-1}$ for the 3 hours irradiated gel. This value remains constant at additional irradiation time. This shows that upon contraction, and counterintuitively, the mesh of the network increases in size and remains constant only after $3 \mathrm{~h}$ of light exposure.

Table 3. Evolution of the mesh size of the network with $c / c^{*}$ before and after contraction obtained after fitting the data with eq 11 . Values obtained for the radius of gyration of the heterogeneities, $R_{g}$, are outside the neuron length scale and should be considered as a lower bound.

\begin{tabular}{|c|c|c|c|c|c|}
\hline $\begin{array}{c}M_{\mathrm{w}}(\mathrm{PEG}) \\
\mathrm{g} / \mathrm{mol})\end{array}$ & $c / c^{*}$ & \multicolumn{2}{|c|}{ Before Irradiation } & \multicolumn{2}{c|}{ After irradiation } \\
\hline & & $\xi(\mathrm{nm})$ & $R_{g}(\mathrm{~nm})$ & $\xi(\mathrm{nm})$ & $R_{g}(\mathrm{~nm})$ \\
\hline 3000 & 0.43 & $11.2 \pm 0.4$ & 75 & $15 \pm 0.4$ & 88 \\
\hline 5000 & 0.78 & $12.3 \pm 0.4$ & 48 & $16 \pm 0.6$ & 50 \\
\hline 5000 & 1 & $5.2 \pm 0.2$ & 59 & $9.7 \pm 0.3$ & 60 \\
\hline 4790 & 1.28 & $3.3 \pm 0.3$ & 55 & $4.5 \pm 0.4$ & 60 \\
\hline 10000 & 1.71 & $5.2 \pm 0.3$ & 45 & $6.1 \pm 0.2$ & 50 \\
\hline 5000 & 1.97 & $4.3 \pm 0.2$ & 35 & $5.9 \pm 0.4$ & 40 \\
\hline 20000 & 2.9 & $5.4 \pm 0.4$ & 50 & $5.7 \pm 0.3$ & 50 \\
\hline 10000 & 3.25 & $5 \pm 0.2$ & 55 & $5 \pm 0.3$ & 55 \\
\hline
\end{tabular}

Data have been fitted according to the already described model (Equation 11) and the obtained results are collected in Table 4 and Figure 8.

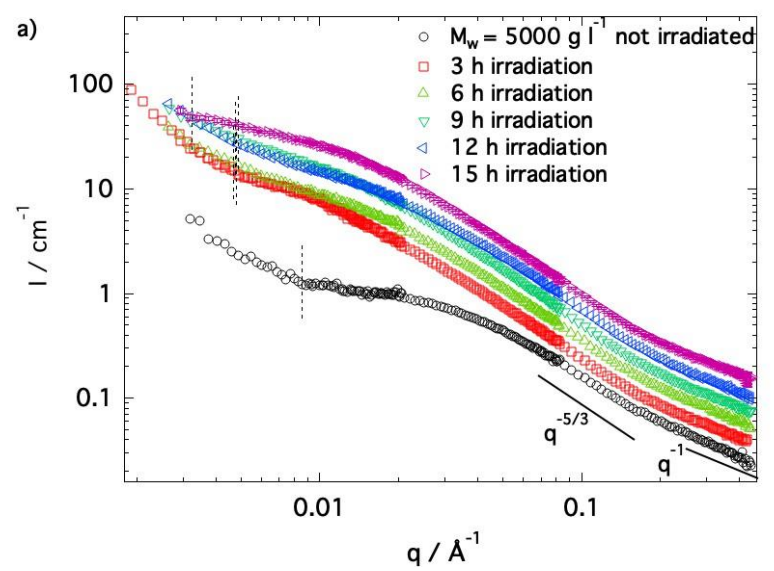




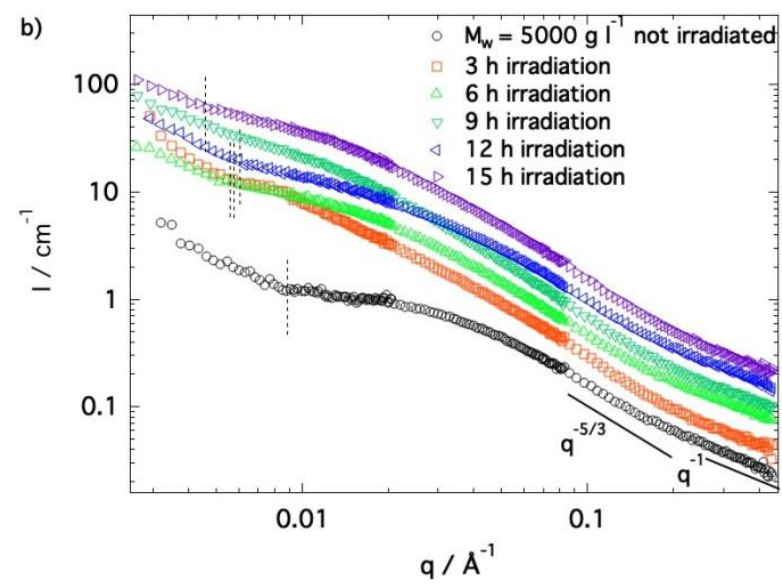

Figure 7. Evolution with irradiation time of the SANS patterns. Figures $7 \mathrm{a}$ and $7 \mathrm{~b}$ represent two sets of experiments showing the reproducibility $\left(M_{\mathrm{W}, \mathrm{PEG}}=5000 \mathrm{~g} \mathrm{~mol}^{-1}, c=\mathrm{c}^{*}=55 \mathrm{~g} / \mathrm{l}\right)$. The dashed lines indicate the onset, $\mathrm{q}^{*}$ upturn, of the low $q$ upturn. For clarity, curves are vertically shifted with respect to each other.

The mesh size of the network increases from $3.7 \mathrm{~nm}$ to $\sim 9 \pm 3 \mathrm{~nm}$ for irradiation times greater than or equal to 3 hours. This value remains constant with further irradiation suggesting that the final local arrangement is reached after only 3 hours of illumination. Instead, the low- $q$ signal associated with the heterogeneities changes continuously during irradiation and the onset of the upturn is gradually shifted toward lower $q$ values before being outside the neutron scattering length scale at maximum contraction. A well-defined plateau associated to the finite mass of the mesh of the network is only visible for $q \leq 0.003 \AA^{-1}$ after $15 \mathrm{~h}$ irradiation. For lower irradiation times, the signal of heterogeneities is mixed with the Lorentzian variation preventing it to converge to a well-defined low- $q$ plateau. The Lorentzian law and thus its contribution to the signal at low- $q$ remains constant for irradiation times lying between $3 \mathrm{~h}$ and $15 \mathrm{~h}$, suggesting that the shift of the onset of the upturn toward lower $q$-values is due to an increase in size of the heterogeneities. The irradiation time evolution of the upturn onset $q^{*}$ upturn is summarized in Figure 9. While the correlation length remains constant after $3 \mathrm{~h}$ irradiation with an increase that may be limited by the rate of possible windings/loops of chain pairs between heterogeneities, the value of the onset of the upturn, $q^{*}$ upturn, decreases over the whole range of irradiation times. The final contracted gel is thus characterized by both a larger network mesh and larger heterogeneities.

\begin{tabular}{|c|c|c|}
$\begin{array}{c}\text { Irradiation time / } \\
\mathrm{h}\end{array}$ & $\begin{array}{c}\text { Mesh size, } \xi / \\
\mathrm{nm}\end{array}$ & Volume ratio $V_{\mathrm{i}} / V_{\mathrm{f}}$ \\
\hline 0 & 3.7 & 1 \\
\hline 3 & 13 & 1.37 \\
\hline 6 & 8 & 1.47 \\
\hline 9 & 9 & 1.52 \\
\hline 12 & 7 & 1.54 \\
\hline 15 & 9.7 & 1.82 \\
\hline
\end{tabular}

Table 4. Evolution with irradiation time of the mesh size of the polymeric network and of the ratio between non-irradiated and irradiated gel volumes $V_{\mathrm{i}} / V_{\mathrm{f}}\left(M_{\mathrm{W}, \mathrm{PEG}}=5000 \mathrm{~g} \mathrm{~mol}^{-1}, c=\mathrm{C}^{*}=55 \mathrm{~g} / \mathrm{l}\right)$.

\section{Behavior of motor aggregation in gels as probed by SAXS}

To further validate the scenario arising from SANS measurements, small angle $x$-ray scattering (SAXS) experiments, which are more sensitive to the total signal of polymer-motor conjugates and thus to the global structure of the gel, were performed. In particular, the contribution of the signal of the motor aggregates (i.e. heterogeneities) to the scattered intensity is higher than in SANS and is thus expected to remain strong in the low- $q$ domain and not be masked anymore by the Lorentzian law associated to the signal of the PEG network. This is what is observed in Figure 10 reporting the SAXS profiles obtained before and after irradiation for different concentrations spanning both regimes $c<c^{\star}$ and $c>$ $C^{\star}$ and different PEG molecular weights. At first sight, the SAXS experimental curves display an overall behavior which seems similar to that obtained in SANS with a $q^{3}$ low- $q$ upturn associated with the signal of gel heterogeneities. Although, we may still observe the intermediate excluded volume regime 
in which the dependence of the scattered intensity is relatively close to a power law with the characteristic exponent of $-5 / 3$, the "better" mixing of the signal of the two populations, polymer network and motors, prevent us to clearly distinguish the high- $q$ rod-like regime. As for the Lorentzian variation, it is almost masked in all patterns. At high $q$, a variation similar to a Guinier one and which may be associated to the finite size of individual motors is now visible, especially for the $c / c^{*}=1$ spectrum. The signal mixing smoothes the curves and complicates their analysis. However, the most noticeable result concerns the well-defined low- $q$ upturn, which is extended towards larger $q$ values (particularly in Figures $5 \mathrm{a}$ and $5 \mathrm{~b}$ ), observed in all spectra and, more importantly, which is identical before and after irradiation even for $c / c^{*}=1$. This, unambiguously, indicates that heterogeneities do not disappear under contraction of the gel. Hence, SAXS confirms the hypothesis expressed previously and based on the increase of the heterogeneities under contraction through the SANS $q^{*}$ upturn analysis.

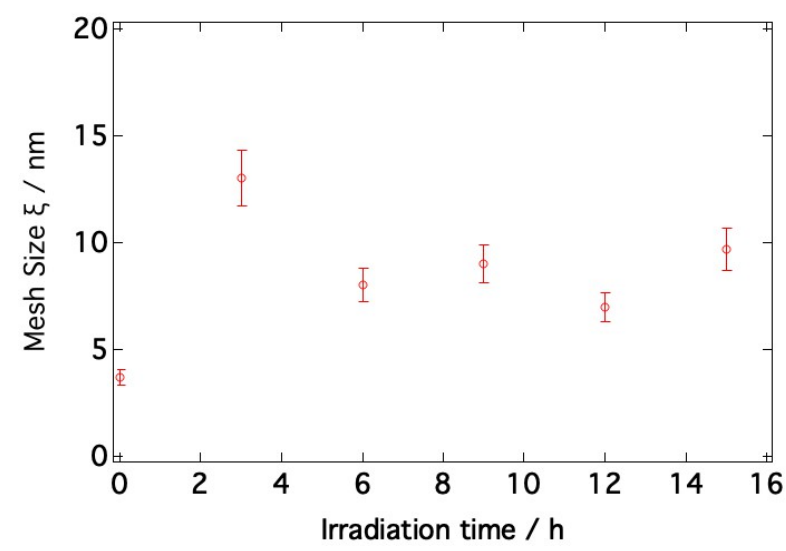

Figure 8. Evolution of the mesh size of the network, $\xi$, with irradiation time for $c / c^{*}=1\left(M_{\mathrm{W}, \mathrm{PEG}}=5000 \mathrm{~g} / \mathrm{mol}, c=55 \mathrm{~g} / \mathrm{l}\right)$.

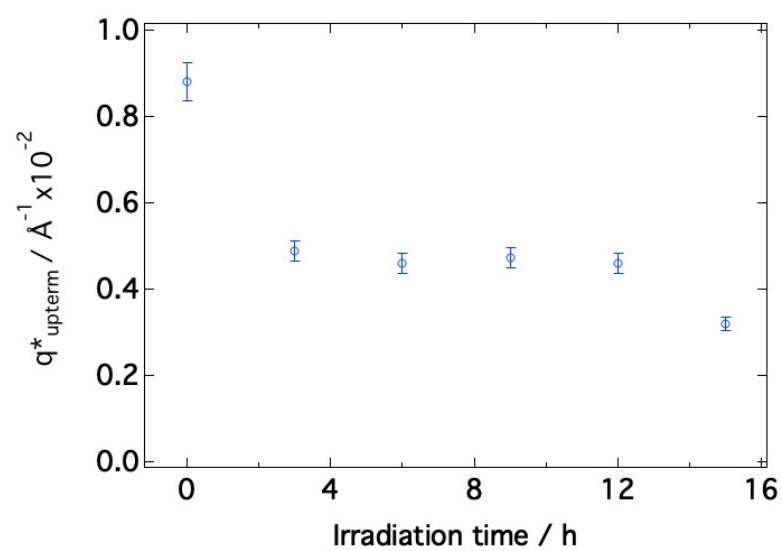

Figure 9. Evolution of the onset of the low- $q$ upturn, $q^{*}$ upturn, with irradiation time (data from Figure $\left.7 \mathrm{a}\right)$ for $c / c^{*}=1\left(M_{\mathrm{W}, \mathrm{PEG}=5000}\right.$ $\left.\mathrm{g} \mathrm{mol}^{-1}, c=55 \mathrm{~g} / \mathrm{l}\right)$.

The rotation of individual effective motors between such domains induces rapprochements and mergers of heterogeneities by winding of the bridging chains. These motors are then eaten up and their increasing number inside heterogeneities causes the ending of the contraction process since they become non-efficient when incorporated in such highly compact domains. The other motors allow winding and twisting of pairs of chains composing the polymeric network; i.e., the matrix. After few hours, the number of individual effective motors is not enough to further contract the denser network. If the system is irradiated for longer times macroscopic damages appear.

Finally, the time-dependent contraction has been followed by SAXS for $c / c^{*}=1$ during 3 hours in order to compare the results with the SANS ones and to observe the process for the global structure. Inspection of the results depicted in Figure 11 shows no changes with irradiation time. All scattering patterns superimpose on each other, indicating that changes are only visible with SANS, a technique more sensitive to the polymer network. In SAXS, the signal of such polymer network and his associated changes (Lorentzian law associated to the mesh of the gel) are masked by the signal of motor 
aggregates (i.e. heterogeneities). Note that the signal of the motors does not change upon irradiation/contraction even if they are efficient; i.e. part of the network.

\section{Estimates of efficient (in network) and non-efficient (in heterogeneities) polymer strands densities}

As for trapped entanglements, loops, dangling chain ends, and other connectivity defects, chains that are in the dense heterogeneities are elastically ineffective strands that do not contribute to the rigidity of the gel. To establish the link between the presence of such heterogeneities and the efficiency of the contraction of the gel, especially at $c^{*}$, it is essential to estimate the density of effective strands ${ }^{40,43-47}$ (i.e., parts of chains between efficient motors in the polymeric network) and thereby the density of ineffective strands into heterogeneities. Formation of heterogeneities is usually inherent to gel formation and makes it difficult to estimate the density of cross-links and thus of polymer strands. ${ }^{44}$ Let us remember that a polymer strand is a chain segment between two cross-links, which are here rotary motors but also classical entanglements and in particular new entanglements appearing during the twisting of the chains under irradiation.

For this purpose, we may use models derived for classical ideal swollen gels and consequently assume that the change of volume is related to the amount of solvent within the gel under contraction (this is of course an approximation because irradiated and non-irradiated gels have different equilibrium swellings). With this assumption $V_{0} \phi_{0}=V \phi=V_{\text {dry }}$, where $\phi_{0}$ is the polymer volume fraction in the preparation state where cross-linking (i.e. click reaction) was performed and $\phi$ the volume fraction of interest in the swollen or partly swollen state. $V_{0}$ and $V$ are the corresponding volumes. On swelling, network polymer strands are stretched as crosslinks, and hence the rotary motors, move apart. Here similarly, irradiation induces the rotation of motors that get closer to each other and induces the de-swelling of the gel.

A general form for the elastic free energy of a swollen or deformed network strand is given by the so-called Panyukov model: 45

$F_{e l} \cong k T \frac{\left(\lambda R_{0}\right)^{2}}{R_{r e f}^{2}}$

Where $\lambda=\left(V / V_{0}\right)^{1 / 3}=\left(\phi_{0} / \phi\right)^{1 / 3}$ is the linear deformation, $R^{2}=\left(\lambda R_{0}\right)^{2}$ the mean-square end-to-end distance of network strands in the final state, which could be the equilibrium or a swollen (here de-swollen) state, and $R_{r e f}^{2}$ the meansquare fluctuation of the end-to-end distance of the network strand that in many cases is equal to the meansquare end-to-end distance of a free chain with the same number of monomers as the strand in the same solution (here the PEG mass between motors). ${ }^{45}$ The effective chain number density, $v=\phi / n b^{3}$, where $n$ is the number of monomer units of size $b$ (i.e. the Kuhn length) in a strand, was estimated using the measurement of the elastic modulus of the gel. ${ }^{30}$ For an ideal gel, the latter depends on the swollen state (i.e. on $\phi$ ) and represents the product of $\phi / n b^{3}$ and $F_{\text {el. }}$ In good solvent we have ${ }^{43,45}$

$$
\begin{aligned}
& G(\phi) \cong k T \frac{\phi}{n b^{3}}\left(\frac{\lambda R_{0}}{R_{r e f}}\right)^{2} \cong k T \frac{\phi}{n b^{3}}\left(\frac{\phi_{0}}{\phi}\right)^{2 / 3}\left(\frac{\phi}{\phi_{0}}\right)^{(2 v-1) /(3 v-1)} \\
& \cong \frac{k T}{n b^{3}} \phi_{0}^{1 /[3(3 v-1)]} \phi^{(9 v-4) /[3(3 v-1)]}
\end{aligned}
$$

Valid for $1 / Q<\phi_{0}<\phi^{* *}$ with the swelling ratio $Q=V_{e q} / V_{\text {dry }}\left(V_{\text {eq }}\right.$ is the volume in the fully swollen state and $V_{\text {dry }}$ the volume in the dry state), $\phi^{* *} \approx v / b^{3}$ the crossover concentration between semidilute and concentrated solutions, and $v=0.588(-1 / v=-5 / 3$ as shown by SANS experiments).
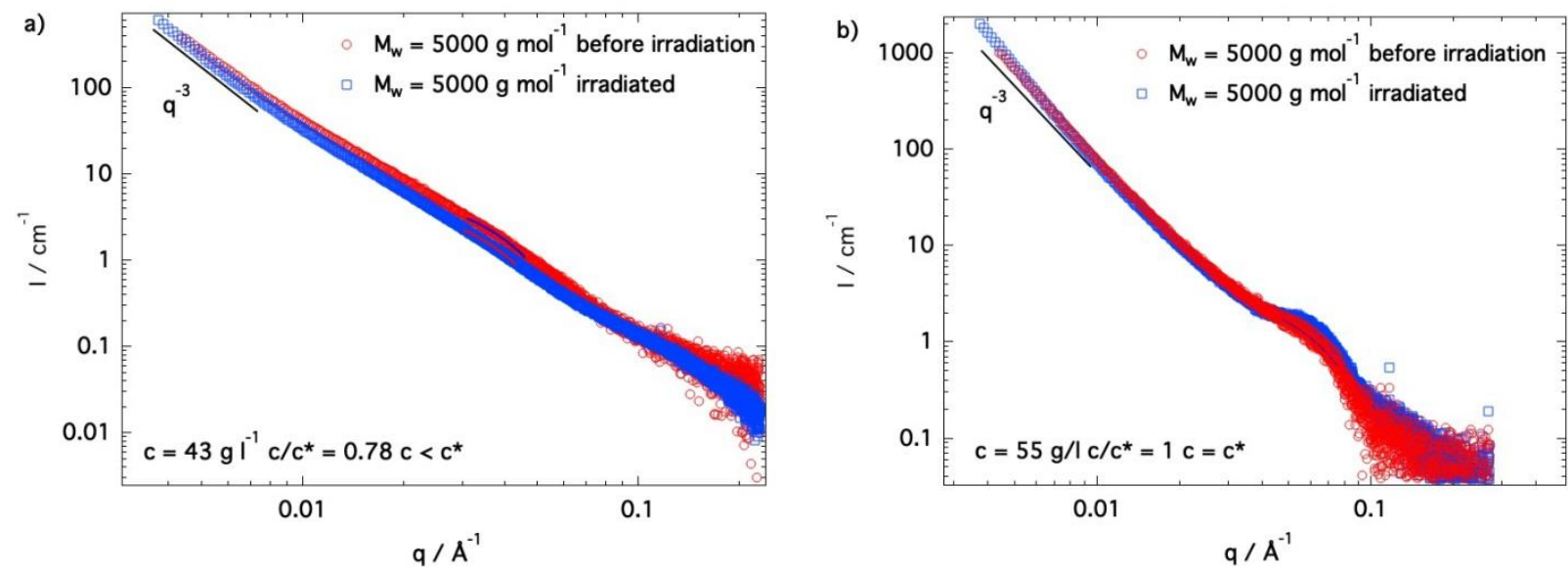

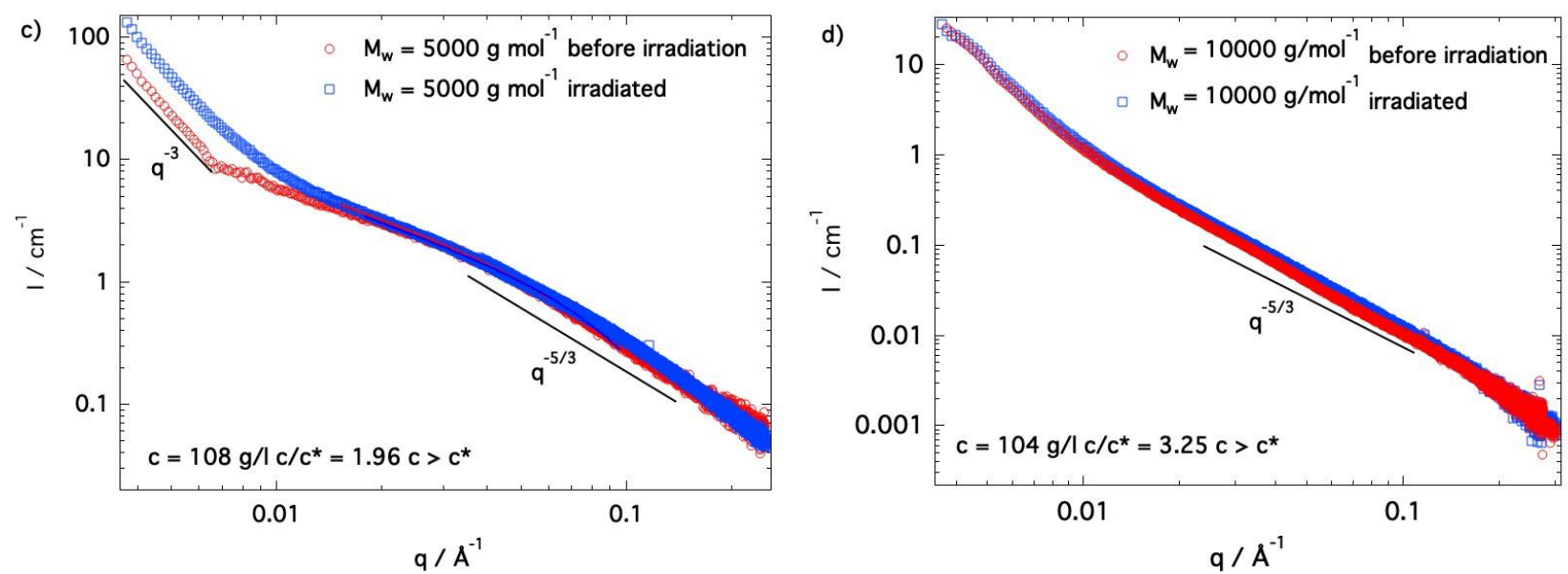

Figure 10. SAXS profiles obtained before and after irradiation for $c / c^{*}=0.78$ (a); 1 (b); 1.96 (c); and 3.25 (d). 


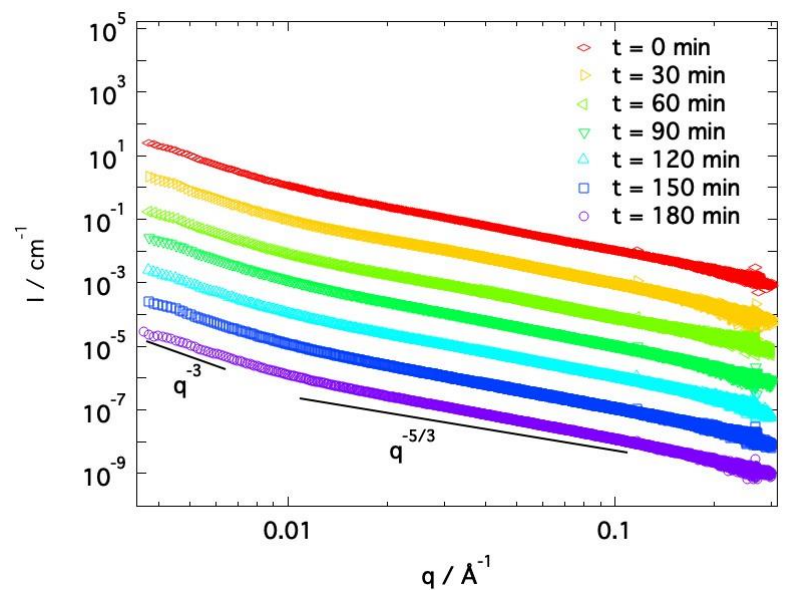

Figure 11. Evolution with irradiation time of the SAXS profiles for $c / c^{*}=1\left(c=\ldots g / l\right.$ and $M w, P E G=10000$ gmol $\left.^{-1}\right)$. Curves are vertically shifted with respect to each other for clarity.

Using such model (Equation 13a), we obtained at $c / c^{*}=1\left(M_{\mathrm{W}, \mathrm{PEG}}=5000 \mathrm{~g} \mathrm{~mol}^{-1}, N=110, c=55 \mathrm{~g} / \mathrm{l}\right)$ a number density of effective polymer strands before irradiation equal to vnon-irradiated $=\left(\phi / n b^{3}\right)_{\text {non-irradiated }} \cong$ $1.65 \times 10^{24}$ strands $/ \mathrm{m}^{3}$ (where $\phi=\phi 0$ and $G^{\prime}$ non-irradiated $=6800 \mathrm{~Pa}$ ), which is much smaller than the polymer density used for the preparation of the gel $\left(6.1 \times 10^{24} \mathrm{PEG}\right.$ chains $\left./ \mathrm{m}^{3}\right)$. This significant outcome indicates that a large part of the material is within the heterogeneities; i.e. $4.45 \times 10^{24}$ strands $/ \mathrm{m}^{3}$, assuming that $n$ is initially equal to the polymerization degree $N=110$ of a PEG chain between two motors.

To estimate the number density of efficient polymer strands in the network after irradiation for the contracted gel, Virradiated, one may use the value of $\phi$ non-irradiated instead of the total preparation volume

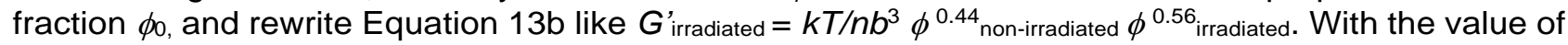
$G_{\text {irradiated }}=20527 \mathrm{~Pa}$ determined in Reference 34, one obtains an order of magnitude (using $n=110$ ) for the final number density irradiated $=\left(\phi / n b^{3}\right)$ irradiated equal to $1.18 \times 10^{25} \mathrm{strands} / \mathrm{m}^{3}$, a value $\sim 7$ times larger than the initial one, which in any case is larger than $V_{\text {non-irradiated }} / V_{\text {irradiated }} \sim 3$ and indicates the formation of new entanglements in the network. This is a direct experimental observation at nanoscale that the motors rotation indeed twists pairs of neighboring polymer chains leading to the formation of new entanglements which play the role of additional cross-links in the network and are responsible of the elastic modulus increase. Note that the number of PEG units between crosslinks (motors and new entanglements between pairs of chains), $n$, is reduced during contraction and should be well below 110 (see Figure 12).

Because the winding of the polymer chains leaves more space between the newly entangled pairs, it increases the averaged mesh size of the network despite the contraction of the material by a factor $\sim 3$ at $c^{\star}$. At the same time heterogeneities grow in size due to rapprochements. Figure 12 shows a picture of the contraction process for $c / c^{*}$. For larger concentrations, in the $c>c^{*}$ regime, additional entanglements become detrimental to the winding of the chains under motor rotation and, therefore the contraction efficiency is well reduced. 


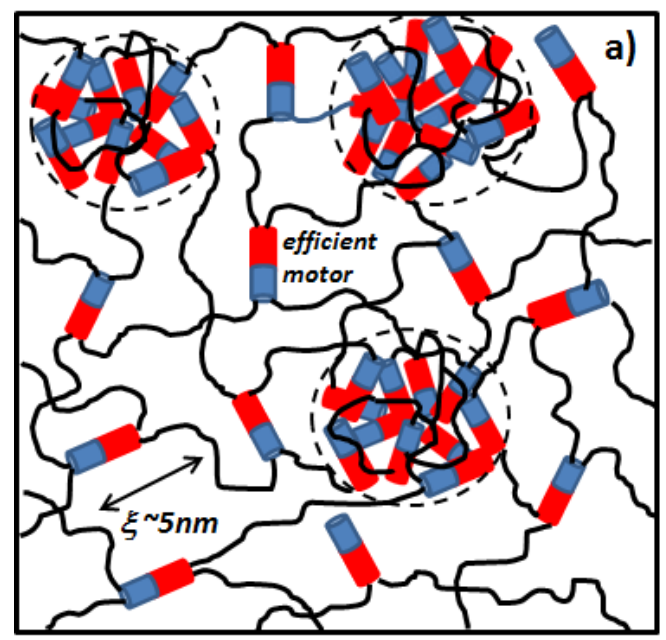

Volume contraction 3 at $c^{*}$

Linear deformation $\lambda \sim 1.4$

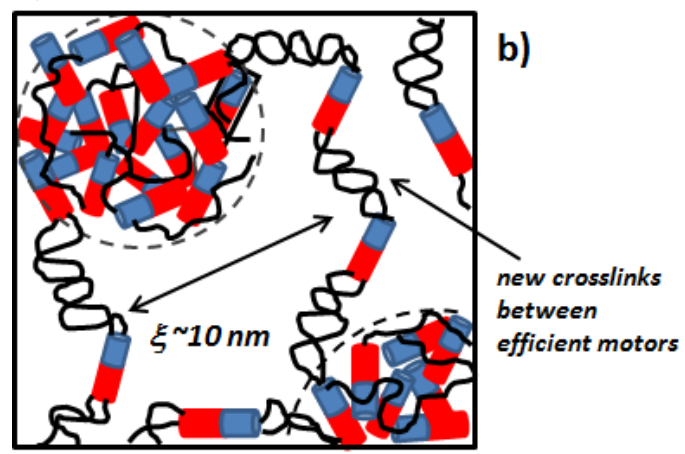

Figure 12. Gel before (a) and after irradiation (b) at $c / c^{*} \sim 1$. Under irradiation, the rotation of efficient motors introduces twisting of chain pairs and new entanglements causing the increase of the number of PEG strands and of the rigidity of the contracted material. Concomitantly, the sizes of the mesh size of the network and of heterogeneities increase.

\section{Conclusions}

By combining SANS and SAXS experiments, we have revealed the importance of samples preparation and of heterogeneities in the contraction efficiency of the first generation of motorized active gels. In particular, we have highlighted an optimal value of 1 for the characteristic ratio, $c / c^{*}$, which was tuned by changing the concentration and/or the polymer length between crosslinks. Importantly, this optimal $c / c^{*}=1$ ratio can be obtained with various value pairs for the chain length and concentration, permitting the maximization of the number of active reticulation units and offering a future wide range of conditions of preparation to potentially access more efficient active materials.

In addition, variations in size of the mesh of the network and of heterogeneities were followed as a function of irradiation time, concentration, and polymer length using in-depth scattering experiments under UV-irradiation. The mesh is found to increase in size for all $c / c^{*}$ values ranging between 0.43 and 3 . This increase is also found to be strongly correlated with the contraction efficiency and is maximum for $c / c^{*}=1$. For values larger than 3 , the higher compacity of the material is detrimental for the twisting of the polymer chains under motor rotation and for a significant macroscopic contraction. At $c / c^{*}=1$, the chains twisting produces simultaneously the maximal increase in correlation length and in size of heterogeneities. The tracking of the structural changes within the gel with irradiation times identified two regimes at the following time-scales: (i) a first time-range (of $3 \mathrm{~h}$ in the present experimental conditions) revealing the saturation of the increase of the mesh of the network, and (ii) a longer time-range (here of $15 \mathrm{~h}$ ) showing the continuous growth of heterogeneities due to rapprochements and/or absorption of neighboring motors. Integrated motions of the molecular motors are also observed over several orders of magnitude, from the nanometric variation of the size of the mesh of the network to the macroscopic contraction of the gel.

Finally, although heterogeneities are found to produce elastically ineffective strands and to be a parameter of major importance to improve the efficiency of such active materials, the macroscopic contraction is a direct consequence of the motorized polymer chains twisting. The 7 times increase in the number of efficient polymer strands during the rotation/contraction process may be related to an 
energy which is proportional to the number of new strands times the elastic energy per chain. It confirms that this type of motorized system can convert light energy toward elastic energy. The improvement of the gel homogeneity by future chemical designs, as used for classical swollen gels, ${ }^{46,47}$ is then of particular interest to enhance the efficiency of active polymeric materials implementing molecular motors.

\section{Conflicts of interest}

There are no conflicts to declare.

\section{Acknowledgements}

This work was supported by the Agence Nationale pour la Recherche (Grant number ANR-14-CE060021, fellowship to G. M.). We also wish to thank the Institut Laue Langevin (ILL-Grenoble, France), the Laboratoire Léon Brillouin (LLB-Saclay, France), and the Soleil Synchrotron Facility (Saint-Aubin, France) for beam-time allocations. We would also like to express our sincere thanks to our local contacts, R. Schweins (ILL), S. Prevost (ILL), F. Cousin (LLB), and J. Pérez (Soleil), as well to all the staff of D11, PA20, and SWING beamlines for their precious help during the SANS and SAXS experiments.

\section{Notes and references}

1 E. Moulin, L. Faour, C. C. Carmona-Vargas, N. Giuseppone, Adv. Mat. 2019, DOI: 10.1002/adma.20190636.

2 M. Schliwa and G. Woehlke, Nature, 2003, 422, 759-765.

3 R. D. Vale and R. A. Milligan, Science, 2000, 288, 88-95.

4 J. L. Krans, Nat. Educ., 2010, 3, 66-69.

5 D. Dattler, G. Fuks, J. Heiser, E. Moulin, A. Perrot, X. Yao, N. Giuseppone, Chem. Rev. 2019, accepted.

6 R. S. Stoll and S. Hecht, Angew. Chem., Int. Ed., 2010, 49, 5054-5075.

7 G. Du, E. Moulin, N. Jouault, E. Buhler and N. Giuseppone, Angew. Chem., Int. Ed., 2012, 51, 12504-12508.

8 J. Chen, F. K.-C. Leung, M. C. A. Stuart, T. Kajitani, T. Fukushima, E. van der Giessen and B. L. Feringa, Nat. Chem., 2018, 10, 132-138.

9 D. J. van Dijken, J. Chen, M. C. A. Stuart, L. Hou and B. L. Feringa, J. Am. Chem. Soc., 2016, 138, 660-669.

10 Q. Lin, X. Hou and C. Ke, Angew. Chem., Int. Ed., 2017, 56, 4452-4457.

11 Y. Lin, X. Jiang, S. T. Kim, S. B. Alahakoon, X. Hou, Z. Zhang, C. M. Thompson, R. A. Smaldone and C. Ke, J. Am. Chem. Soc., 2017, 139, 7172-7175.

12 A. Goujon, G. Du, E. Moulin, G. Fuks, M. Maaloum, E. Buhler and N. Giuseppone, Angew. Chem., Int. Ed., 2016, 55, 703-707.

13 A. Goujon, G. Mariani, T. Lang, E. Moulin, M. Rawiso, E. Buhler and N. Giuseppone, J. Am. Chem. Soc., 2017, 139, 4923-4928.

14 G. Mariani, A. Goujon, E. Moulin, M. Rawiso, N. Giuseppone, and E. Buhler, Nanoscale, 2017, 9, $18456-18466$.

15 A. Goujon, T. Lang, G. Mariani, E. Moulin, G. Fuks, J. Raya, E. Buhler and N. Giuseppone, J. Am. Chem. Soc., 2017, 139, 14825-14828.

16 L. Gao, Z. Zhang, B. Zheng and F. Huang, Polym. Chem., 2014, 5, 5734-5739.

17 A. Wolf, E. Moulin, J. J. Cid Martín, A. Goujon, G. Du, E. Busseron, G. Fuks and N. Giuseppone, Chem. Commun., $2015,51,4212-4215$.

18 K. Iwaso, Y. Takashima and A. Harada, Nat. Chem., 2016, 8, 625-632.

19 M. Nakahata, Y. Takashima, H. Yamaguchi and A. Harada, Nat. Commun., 2011, 2, 511.

20 Y. Yu, M. Nakano and T. Ikeda, Nature, 2003, 425, 145.

21 Y. Yu, T. Maeda, J. Mamiya and T. Ikeda, Angew. Chem., Int. Ed., 2007, 46, 881-883.

22 S. Kassem, T. van Leeuwen, A. S. Lubbe, M. R. Wilson, B. L. Feringa, and D. A. Leigh, Chem. Soc. Rev. 2017, 46, 2592.

23 M. Baroncini, S. Silvi, and A. Credi, Chem. Rev. 2019, DOI:10.1021/acs.chemrev.9b00291.

24 N. Koumura, R. W. J. Zijlstra, R. A. van Delden, N. Harada and B. L. Feringa, Nature, 1999, 401, $152-155$.

25 B. L. Feringa, Angew. Chem., Int. Ed., 2017, 56, 11060-11078.

26 R. Eelkema, M. M. Pollard, J. Vicario, N. Katsonis, B. S. Ramon, C. W. M. Bastiaansen, D. J. Broer and B. L. Feringa, Nature, 2006, 440, 163.

27 Q. Li, G. Fuks, E. Moulin, M. Maaloum, M. Rawiso, I. Kulic, J. T. Foy and N. Giuseppone, Nat. Nanotechnol., 2015, 10, 161-165.

28 Q. Li, J. T. Foy, J.-R. Colard-Itté, A. Goujon, D. Dattler, G. Fuks, E. Moulin and N. Giuseppone, Tetrahedron, 2017, 73, 4874-4882.

29 J. T. Foy, Q. Li, A. Goujon, J.-R. Colard-Itté, G. Fuks, E. Moulin, O. Schiffmann, D. Dattler, D. P. Funeriu and N. Giuseppone, Nat. Nanotechnol., 2017, 12, 540-545.

30 J.-R. Colard-Itté, Q. Li, D. Collin, G. Mariani, G. Fuks, E. Moulin, E. Buhler and N. Giuseppone, Nanoscale, 2019, 11, 5197-5202.

31 E. Buhler, S. J. Candau, J. Schmidt,Y. Talmon, E. Kolomiets and J.-M. Lehn, J. Polym. Sci.: Part B: Polymer Physics, 2007, 45, 103-115. 
32 G. Mariani, E. Buhler, J.-R. Colard-Itté, N. Giuseppone, E. Moulin and R. Schweins (2016). Structural Investigation of Contractile Polymeric Materials based on Light-Activated Rotary Motors. Institut Laue-Langevin (ILL) doi:10.5291/ILL-DATA.9-11-1791.

33 G. Mariani, E. Buhler, J.-R. Colard-Itté, N. Giuseppone, E. Moulin and R. Schweins (2017). Structural studies of covalent polymers decorated with triarylamine units to achieve light-induced folding. Institut Laue-Langevin (ILL) doi:10.5291/ILL-DATA.9-11-1829.

34 G. Mariani, E. Buhler, J.-R. Colard-Itté, D. Dattler, N. Giuseppone, E. Moulin and S. Prevost (2019). Effects of polymer molecular weight on the structure and on the efficiency of light-driven contractile gels. Institut LaueLangevin (ILL) doi:10.5291/ILL-DATA.9-11-1845.

35 M. J. Chmielewski, E. Buhler, J. Candau and J.-M. Lehn, Chem. Eur. J., 2014, 20, 6960-6977.

36 J. Appell, G. Porte and E. Buhler, J. Phys. Chem. B, 2005, 109, 13186-13194.

37 E. Buhler and F. Boué Eur. Phys. J., E 2003, 10, 89-92.

38 E. Buhler and F. Boué, Macromolecules, 2004, 37, 1600-1610.

39 P. G. de Gennes, Scaling Concepts in Polymer Physics, Cornell University Press, Ithaca, 1979.

40 S. P. Obukhov, M. Rubinstein and R. H. Colby, Macromolecules, 1994, 27, 3191-3198.

41 M. Shibayama, T. Tanaka and C. C. Han, J. Chem. Phys. 1992, 97, 6829.

42 S. Mallam, F. Horkay, A.-M. Hecht, A. R. Rennie and E. Geissler, Macromolecules, 1991, 24, 543-548.

43 M. Rubinstein and R. H. Colby, Polymer physics, Oxford University Press, Oxford, New York, 2003.

44 T. Katashima, U. Chung and T. Sakai, Macromol. Symp., 2015, 358, 128-139.

45 S. V. Panyukov, Sov. Phys. JETP, 1990, 71, 372-379.

46 M. Shibayama, Macromol. Symp., 2017, 372, 7-13.

47 T. Sakai, T. Matsunaga, Y. Yamamoto, C. Ito, R. Yoshida, S. Suzuki, N. Sasaki, M. Shibayama and U. Chung, Macromolecules, 2008, 41, 5379-5384. 